\begin{tabular}{||l|l|}
\hline \hline Citation & $\begin{array}{l}\text { A. Caiazzo, N. Alujevic, B. Pluymers and W. Desmet, (2018) } \\
\text { Active control of turbulent boundary layer-induced sound transmission } \\
\text { through the cavity-backed double panels } \\
\text { Journal of Sound and Vibration, 422, 161-188. }\end{array}$ \\
\hline Archived version & $\begin{array}{l}\text { Author manuscript: the content is identical to the content of the published } \\
\text { paper, but without the final typesetting by the publisher. }\end{array}$ \\
\hline Published version & https://www.sciencedirect.com/science/article/pii/S0022460X18301172 \\
\hline Journal homepage & http://www.sciencedirect.com/science/journal/0022460X \\
\hline Author contact & $\begin{array}{l}\text { Anna.Caiazzo@kuleuven.be } \\
+32(0) 16372048\end{array}$ \\
\hline
\end{tabular}

(article begins on next page) 


\title{
Active control of turbulent boundary layer-induced sound transmission through the cavity-backed double panels
}

\author{
A. Caiazzo ${ }^{\mathrm{a}, \mathrm{b}, *}$, N. Alujevića ${ }^{\mathrm{a}}, \mathrm{B}$. Pluymers ${ }^{\mathrm{a}, \mathrm{b}}$, W. Desmet $^{\mathrm{a}, \mathrm{b}}$ \\ ${ }^{a}$ KU Leuven, Department of Mechanical Engineering, Celestijnenlaan 300B, 3001 Heverlee, Belgium. \\ ${ }^{b}$ Member of Flanders Make.
}

\begin{abstract}
This paper presents a theoretical study of active control of turbulent boundary layer (TBL) induced sound transmission through the cavity-backed double panels. The aerodynamic model used is based on the Corcos wall pressure distribution. The structural-acoustic model encompasses a source panel (skin panel), coupled through an acoustic cavity to the radiating panel (trim panel). The radiating panel is backed by a larger acoustic enclosure (the back cavity). A feedback control unit is located inside the acoustic cavity between the two panels. It consists of a control force actuator and a sensor mounted at the actuator footprint on the radiating panel. The control actuator can react off the source panel. It is driven by an amplified velocity signal measured by the sensor. A fully coupled analytical structural-acoustic model is developed to study the effects of the active control on the sound transmission into the back cavity. The stability and performance of the active control system are firstly studied on a reduced order model. In the reduced order model only two fundamental modes of the fully coupled system are assumed. Secondly, a full order model is considered with a number of modes large enough to yield accurate simulation results up to $1000 \mathrm{~Hz}$. It is shown that convincing reductions of the TBL-induced vibrations of the radiating panel and the sound pressure inside the back cavity can be expected. The reductions are more pronounced for a certain class of systems, which is characterised by the fundamental natural frequency of the skin panel larger than the fundamental natural frequency of the trim panel.
\end{abstract}

Keywords: Turbulent Boundary Layer, Corcos, Active vibroacoustic control, Direct velocity feedback, Active control of sound transmission, Stability of active systems

\section{Introduction}

In high speed automotive, aerospace, and railway transportation, the turbulent boundary layer (TBL) is one of the most important sources of interior noise. For example, in large passenger aircraft, the principal source of interior noise is the TBL during typical cruise conditions [1, 2]. The stochastic pressure distribution associated with the turbulence is able to significantly excite structural vibrations of vehicle exterior panels. These vibrations are transmitted to the interior

${ }^{*}$ Corresponding author

Email address: Anna. Caiazzo@kuleuven. be (A. Caiazzo) 
trim panels through the structural and acoustical paths between the interior and exterior panels. Consequently the trim panels radiate sound into the vehicle interior.

The TBL excitation is random and broadband. In order to model this excitation, semi-empirical models for the wall pressure distribution are usually used. Between the different models developed over the years to describe the wall pressure fluctuations due to a TBL [3], the Corcos model is among the simplest [4, 5]. This is because the space variables are separated and the phase variation is only accounted for along the streamwise direction. It should be noted that modelling the low-wavenumber region of the TBL pressure spectrum is still an active area of research [6, 7]. In particular, it is known that Corcos model overpredicts experimental results in terms of the spatially integrated correlation functions. It nevertheless enables rather realistic representations of the wall pressure distribution at and near the convective wavenumber, where most of the energy in the boundary layer pressure fluctuations is concentrated. Therefore, the Corcos model is often used as a reference for other models [8, 9, 10], such as, for example, the recently developed Generalized Corcos model [7].

The disturbance produced by TBL typically excites the vehicle exterior surface in a broad band of frequencies including the low-frequency range. The resulting transmission of sound into the interior is usually controlled by passive means. However, the effectiveness of passive noise control treatments, such as, for example, sound absorbing materials, is limited to frequencies where the acoustic wavelengths are short, in the medium and high frequency range. As a rule of thumb, the sound absorbing layers should be at least as thick as $1 / 4$ of the acoustic wavelength in order to absorb the sound effectively. The passive sound transmission control is ineffective in the lowfrequency range since the passive sound absorptive materials can not attenuate the large-length waves. On the other hand, double leaf partitions may be used whose transmission loss increases more rapidly with frequency than that of single leaf partitions. Therefore, it is often the case that the vehicles are equipped with additional interior trim panels. In such arrangements, the sound absorbing layers are packed in the space available between the two panels. Even with such integrated approach, the transmission loss of double leaf partitions is still rather poor at the frequencies below the mass-air-mass resonance [11, 12].

For these reasons, interest has grown in investigating active control of low-frequency sound transmission through double panels [13, 14]. Active control of sound transmission can be used both with deterministic and stochastic disturbances. Feed-forward and feedback active control systems have been considered in this sense. Although feed-forward control methods are more easily applied in situations with deterministic, tonal disturbances [15, 16], it is also possible to use them to control noise of stochastic origin [17]. This, however, requires that creative arrangements are used to obtain the reference signals well correlated to the disturbance [18, 19]. Here a good balance between the causality margin and the coherence with respect to the reference and disturbance signals is essential [19].

Feedback control systems can be used instead, and some promising results have been reported in the last decade [14, 20, 21, 22]. The use of feedback control is appealing since the reference signals are not necessary. In this context, some very effective feedback control methods have been proposed for vibration isolation [23, 24, 25]. These include, for example, the so-called skyhook damping and integral force feedback [26, 27]. Such methods can overcome the difficulties with traditional passive vibration isolation systems. The difficulties include the intrinsic trade-off in the vibration isolation performance between the low-frequency resonance-controlled transmission and the high-frequency mass-controlled transmission. With active vibration isolation the low frequency vibration transmission may be attenuated without compromising the high-frequency roll-off of the vibration transmissibility with frequency. Such active vibration isolation methods 
have been typically considered in the situations where the mechanical systems at hand can be accurately represented through their lumped parameter approximation [23, 24, 25].

In this paper, it is shown that feedback vibration isolation techniques can be very effective to actively control the sound transmission in distributed parameter systems with complex structuralacoustic coupling. In particular, the TBL-induced sound transmission from a flexible panel (the source panel) through an acoustic medium to another flexible panel (the radiating panel) and further into additional acoustic enclosure, is treated as an active vibration isolation problem. Thus the problem studied roughly approximates the problem of the transmission of aerodynamic noise into high-speed vehicles. The stability and performance of the control system based on direct velocity feedback, are addressed in detail. It is shown that they are substantially affected by the passive properties of the double panel system before control. Particularly acoustic reductions of the transmitted sound are feasible with a certain class of double panels that are characterised by the fundamental natural frequency of the source panel larger than that of the radiating panel. Then the frequency spectra of sound pressure in the acoustic enclosure approximating the vehicle interior can be successfully attenuated in the low frequency range without an overshot in the high frequency range. As a result, significant broadband sound transmission control can be realised, even though only a single control unit is used.

The paper is structured as follows. In Section 2, an outline of the mathematical model used to couple the aerodynamic, control and vibroacoustic aspects of the problem is given. In Section 3 , the stability and performance of the active control system are discussed on a reduced order model, whereas in Section 4 a full-order model is considered. First a point force excitation with white noise spectral distribution is used as a reference case, and then, the stochastic TBL pressure distribution is considered - both for the reduced and the full order models.

\section{The model problem}

The analytical model outlined in this section is used to predict the noise transmission through an acoustically coupled double panel system into a rectangular cavity when an active vibration isolation unit is used. The problem under analysis is physically given by the interaction of an aerodynamic model, that represents the TBL pressure fluctuations on the structure, and a structural-acoustic model, that gives the noise transmission and interior sound levels.

\subsection{Aerodynamic model}

The wall pressure field, generated by a fully developed TBL with zero mean pressure gradient, can be regarded as homogeneous in space and stationary in time [3, 28]. Under such conditions, for a flow in the $x$-direction over the $(x, y)$ plane, the generated pressure field can be expressed by a cross power spectral density (CPSD) function decaying with spatial and time separations and convected with the flow as follows,

$$
\Psi_{p p}\left(\zeta_{x}, \zeta_{y}, \omega\right)=\phi(\omega) \Psi\left(\zeta_{x}, \zeta_{y}, \omega\right),
$$

in which $\zeta \equiv\left(\zeta_{x}, \zeta_{y}, 0\right)$ is the spatial separation vector, $\phi(\omega)$ is the single-point wall-pressure spectrum (i.e., auto-spectrum) and $\Psi\left(\zeta_{x}, \zeta_{y}, \omega\right)$ is a spatial correlation function, that, according to Corcos, is given by

$$
\Psi\left(\zeta_{x}, \zeta_{y}, \omega\right)=\mathrm{e}^{-\mathrm{i} k_{\omega} \zeta_{x}} \mathrm{e}^{-\left|\zeta_{x}\right| k_{\omega} \alpha_{x}-\left|\zeta_{y}\right| k_{\omega} \alpha_{y}} .
$$

The convective wavenumber is $k_{\omega}=\omega / U_{c}$, given by the angular frequency $\omega$ and the convective velocity $U_{c}=0.7 U_{\infty}$, with $U_{\infty}=240 \mathrm{~m} \mathrm{~s}^{-1}$. In this study the dimensions of the structure and the 
convective speed are fixed. It is thus possible that the results presented in the paper qualitatively differ for different convective speeds and the dimensions of the panels.

In the following sections the properties of the physical system used are defined in detail. In this study, an aerospace application is considered in which the model approximates a rectangularly shaped aircraft multi panel wall. The longitudinal and lateral decay rates of the coherences, $\alpha_{x}$ and $\alpha_{y}$ respectively, are normally chosen to yield good agreement with experiments. In the following analysis, the empirical parameters are chosen as $\alpha_{x}=0.10$ and $\alpha_{y}=0.77$, [29, 30]. In Eq. (1), the single-point wall-pressure spectrum, $\phi(\omega)$, is given by a semi-empirical formula defined by Goody [31],

$$
\phi(\omega)=\frac{3\left(\delta / U_{\infty}\right)^{3}\left(\omega \tau_{\omega}\right)^{2}}{\left[\left(\omega \delta / U_{\infty}\right)^{0.75}+0.5\right]^{3.7}+\left[\left(1.1 R_{T}^{-0.57}\right)\left(\omega \delta / U_{\infty}\right)\right]^{7}},
$$

in which $\delta$ is the boundary layer thickness, $\tau_{\omega}=U_{t}^{2} \rho$ is the wall shear stress, with air density $\rho$, and $R_{T}$ is the Reynolds number dependent factor given by

$$
R_{T}=\frac{U_{\tau}^{2} \delta}{U_{\infty} v},
$$

where $U_{\tau}$ is the friction velocity and $v$ is the kinematic viscosity. Such a model compares well with experimental data over a large range of Reynolds numbers [32] and it is able to describe the essential properties of the single point wall pressure spectrum with a limited numbers of variables.

\subsection{Structural-acoustic model}

In Fig. 1] the structural-acoustic model problem is shown. The model encompasses a cavitybacked homogeneous double panel driven on one side by a stationary TBL. Thus, the acoustic enclosure, $c_{2}$, filled with air, has five rigid walls and one flexible double wall. The two flexible panels are acoustically coupled with the air in cavity $c_{1}$ between them. As shown in Fig. 1] the source panel, $p_{s}$, is excited by the grazing flow. Its vibrations generate sound waves which excite the radiating panel, $p_{r}$. Finally, the vibrations of the radiating panel generate sound waves which are radiated to the acoustic enclosure $c_{2}$.

The active control approach in this study is based on isolating the radiating panel from vibrations coming from the source panel through an active vibration isolation unit. A sensor is placed near the centre of the radiating panel, which is collocated to a force actuator. The actuator generates the control force while reacting against the source panel. In parallel to the actuator, a passive damper is mounted with a damping coefficient $c_{p}$, as seen in Fig. 11. In this paper, idealised sensor-actuator transducers are considered, that is, the sensor-actuator internal dynamics are not taken into account. Real sensor-actuator transducers would certainly exhibit more complex highfrequency behaviour and decrease stability margins of the active control systems considered in the study. However, in this paper the stability and performance characteristics of the active control system, which are independent of the sensor-actuator dynamics, are investigated. Also, by neglecting the dynamics of the transducers, benchmark results can be obtained. Additionally, sensors and actuators would certainly add some mass to the system. However, a miniature voicecoil actuator that can develop an equivalent of 140 grams of peak force (approx. $1.4 \mathrm{~N}$ ) has a total mass (coil and magnet) of only about 6 grams with a stroke of about $3 \mathrm{~mm}$. Furthermore such actuators do not inflict any stiffness between their two terminals like, for example, piezoelectric 
stack actuators.

The actuator force $f_{c}$ is made proportional to the negative velocity of the radiating panel, $v_{p_{r}}$, which is measured by the velocity sensor, so that

$$
f_{c}=-g v_{p_{r}}
$$

where $g$ is the feedback gain. Its dimension is the same as that of the damping coefficient, $\mathrm{N}$ $\mathrm{s} \mathrm{m}^{-1}$. Therefore, there are two damping coefficients, the active damping coefficient $g$ and the passive one $c_{p}$.

In the following section, the structural-acoustic model presented describes the interaction between the vibrating structure and the turbulent flow assuming a one-way interaction, i.e. the influence of the panel vibration on the boundary layer is neglected [33]. Under this assumption, it is possible to model the excitation term for the source panel as a 'blocked pressure', i.e. the wall pressure fluctuations that would be observed on a rigid wall [34]. Further hypothesis on the nature of the turbulent boundary layer are listed in Section 2.1

In order to model the structural-acoustic control problem at hand, the two panel displacements and the two acoustic enclosure pressures are calculated by coupling the wave equations for the two cavities with the governing equations for the two panels [35, 36]. To this end, the modal expansion method is used [37]. Simply supported boundaries of the two panels, and rigid cavity walls are assumed [34, 37].

The rectangular coordinates $(x, y, z)$ are chosen, $z$ being normal to the panel, and $x$ in the direction of mean flow, as seen in Fig. 11 The flexural displacement of the mean surface of the panel at the position $\mathbf{r}$ of coordinates $\left(x, y, L_{c_{1_{z}}}\right)$ in the case of the source panel and $(x, y, 0)$ in the case of the radiating panel at the time instant $t$ is defined by $w_{p_{s}}(\mathbf{r} ; t)$ and $w_{p_{r}}(\mathbf{r} ; t)$, respectively. The acoustic pressure fields at the points $\mathbf{x}$ of coordinates $(x, y, z \geq 0)$ in the case of the cavity $c_{1}$ and $(x, y, z \leq 0)$ in the case of the cavity $c_{2}$ at the time instant $t$ is defined by $p_{c_{1}}(\mathbf{x} ; t)$ and $p_{c_{2}}(\mathbf{x} ; t)$,

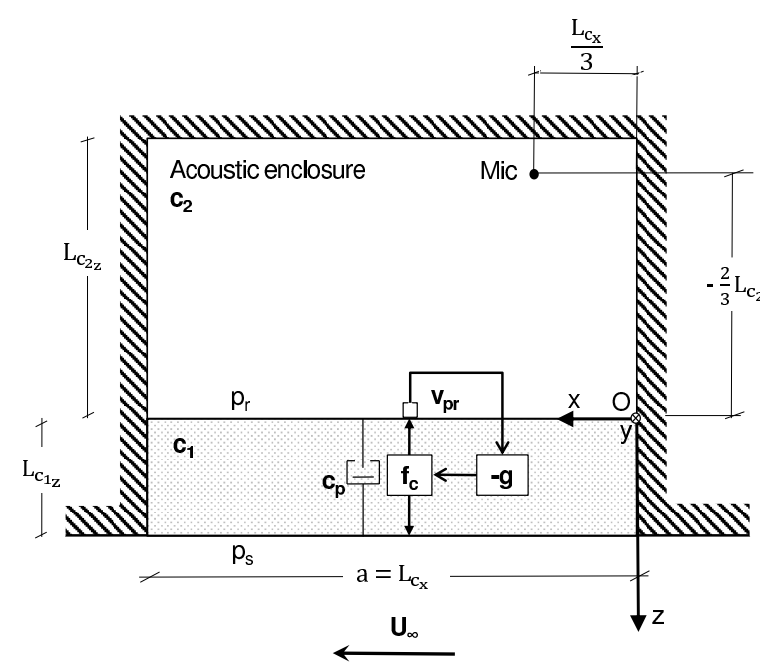

Figure 1: The model problem. 
respectively.

The system equations are given by the combination of the governing equations of the uncoupled systems, which are the plate governing equations for the panels and the inhomogeneous wave equations for the acoustic enclosures. Those are given as follows,

$$
D_{p_{s}} \nabla^{4} w_{p_{s}}+\rho t_{p_{s}} \frac{\partial^{2} w_{p_{s}}}{\partial t^{2}}+\sigma \frac{\partial w_{p_{s}}}{\partial t}=p_{c_{1}}\left(x, y, L_{c_{1}}, t\right)-p_{t b l}\left(x, y, L_{c_{1}}, t\right)+\tilde{f}_{c}+\tilde{f}_{p},
$$

for the source panel $p_{s}$,

$$
D_{p_{r}} \nabla^{4} w_{p_{r}}+\rho t_{p_{r}} \frac{\partial^{2} w_{p_{r}}}{\partial t^{2}}+\sigma \frac{\partial w_{p_{r}}}{\partial t}=p_{c_{2}}(x, y, 0, t)-p_{c_{1}}(x, y, 0, t)-\tilde{f}_{c}-\tilde{f}_{p},
$$

for the radiating panel $p_{r}$,

$$
\nabla^{2} p_{c_{l}}-\frac{1}{c_{0}^{2}} \frac{\partial^{2} p_{c_{l}}}{\partial t^{2}}-\sigma_{a c} \frac{\partial p_{c_{l}}}{\partial t}=-\rho_{0}\left(\frac{\partial v_{p_{r}}}{\partial t}+\frac{\partial v_{p_{s}}}{\partial t}\right),
$$

for the acoustic enclosure $c_{1}$, and

$$
\nabla^{2} p_{c_{2}}-\frac{1}{c_{0}^{2}} \frac{\partial^{2} p_{c_{2}}}{\partial t^{2}}-\sigma_{a c} \frac{\partial p_{c_{2}}}{\partial t}=-\rho_{0} \frac{\partial v_{p_{r}}}{\partial t},
$$

for the acoustic enclosure $c_{2}$. In Eq. (6)-(77), $D_{i}, \rho_{i}, t_{i}$ and $\sigma_{i}$ are the bending stiffness, density, thickness and structural damping ratio of the $i$-th plate, respectively. The right hand side of Eq. (6) consists of the sum of the turbulence pressure, $p_{t b l}(\mathbf{x} ; t)$, which would be observed on a rigid wall and the pressure due to the contribution of the acoustic enclosure $c_{1}, p_{c_{1}}(\mathbf{x} ; t)$. On the other hand, the pressure field on the radiating panel, $p_{r}$, is given by the contribution of the acoustic enclosures $c_{1}$ and $c_{2}$, as shown in Eq. (77). Then, $\tilde{f}_{c}$ and $\tilde{f}_{p}$ are the actuator and damper force per unit area, respectively.

In Eq. (87)-97, $v$ represents the distribution of source volume velocity per unit volume (including the effect of the boundary surface vibration), $c_{0}, \rho_{0}$ and $\sigma_{a c}$ are the sound speed in air, mean density of air and acoustic damping ratio for both cavities, respectively. The right hand side of Eq. (8)-(9) represents a rate of change of mass flux per unit volume, in which $v_{p_{s}}$ and $v_{p_{r}}$ are volume velocities for the source and radiating panel, respectively, which refer to an infinitesimally thin layer situated on a surface just inside a rigid boundary [11].

Subsequently, the system is modelled by expanding the displacements of the source and radiating panels in a series of characteristic functions, that are in-vacuo structural normal modes of a simply supported panel,

$$
w_{i}(\mathbf{r} ; t)=\sum_{m_{x}, m_{y}}^{M_{x_{i}}, M_{y_{i}}} q_{i_{m_{x} m_{y}}}(t) \psi_{i_{m_{x} m_{y}}}(\mathbf{r}), \quad i=\left\{p_{s}, p_{r}\right\} .
$$

In Eq. 10p, $\mathrm{M}_{i}=\mathrm{M}_{x_{i}} \times \mathrm{M}_{y_{i}}$ is the total number of the plate modes. The pressure of the acoustic cavities, $c_{1}$ and $c_{2}$ for the cavity of the double panel system and the back cavity, respectively, are also expanded in terms of the normal modes of a rigid-walled cavity,

$$
p_{c}(\mathbf{x} ; t)=\sum_{n_{x}, n_{y}, n_{z}}^{N_{x c}, N_{y c}, N_{z c}} g_{c_{n_{x} n_{y} n_{z}}}(t) \Gamma_{c_{n_{x} n_{y} n_{z}}}(\mathbf{x}), \quad c=\left\{c_{1}, c_{2}\right\},
$$


where $\mathrm{N}_{c}=\mathrm{N}_{x_{c}} \times \mathrm{N}_{y_{c}} \times \mathrm{N}_{z_{c}}$ is the total number of the enclosure modes.

Because of the Gibbs phenomenon, the summation of the rigid-wall acoustic modes, Eq. (11), does not converge to the correct boundary normal velocity, but does converge correctly to the surface pressure, which is needed for a correct formulation of the coupled equations [11].

In Eq. (10) and (11), $q_{i_{m_{x} m_{y}}}(t)$ and $g_{c_{n_{x} n_{y} n_{z}}}(t)$ are the components of the displacement and pressure time functions, respectively; $\psi_{i_{m_{x} m_{y}}}(x, y)$ and $\Gamma_{c_{n_{x} n_{y} n_{z}}}(x, y, z)$ are the modal functions for the simply supported plate and the rigid body enclosure, respectively, given by

$$
\begin{aligned}
\psi_{i_{m_{x} m_{y}}}(x, y)= & \frac{2}{\sqrt{a b}} \sin \left(m_{x} \pi x / a\right) \sin \left(m_{y} \pi y / b\right), \\
\Gamma_{c_{n_{x} n_{y} n_{z}}}(x, y, z)= & \frac{A_{n_{x}} A_{n_{y}} A_{n_{z}}}{\sqrt{L_{c_{x}} L_{c_{y}} L_{c_{z}}}} \cos \left(n_{x} \pi x / L_{c_{x}}\right) \\
& \times \cos \left(n_{y} \pi y / L_{c_{y}}\right) \cos \left(n_{z} \pi z / L_{c_{z}}\right) .
\end{aligned}
$$

The two plates have the same length $a$ and width $b . L_{c_{x}}, L_{c_{y}}$ and $L_{c_{z}}$ are the acoustic enclosure lengths, widths and heights, respectively, with $c=c_{1}, c_{2}$. In Eq. (13), the constant $A_{n_{x}}, A_{n_{y}}$ and $A_{n_{z}}$ are equal to $\sqrt{2}$ when $n_{x} \neq 0, n_{y} \neq 0, n_{z} \neq 0$ respectively, and, they are equal to 1 when $n_{x}=0, n_{y}=0$ and $n_{z}=0$, respectively [36].

Eq. (10) - (11) are then substituted into the governing equations of motion for the panels and the acoustic enclosures, Eq. (6) - 9). Therefore, making use of the orthogonality properties of the mode shape functions, the complete coupled fluid- structural equations can be written in the matrix form as follows,

$$
\mathbf{M} \frac{\partial^{2} \mathbf{y}}{\partial t^{2}}+\mathbf{D} \frac{\partial \mathbf{y}}{\partial t}+\mathbf{K y}=\mathbf{x}
$$

where $\mathbf{M}, \mathbf{D}$ and $\mathbf{K}$ are the mass, damping and stiffness matrices, given by

$$
\begin{aligned}
\mathbf{M} & =\left[\begin{array}{cccc}
\mathbf{M}_{p_{s} p_{s}} & \mathbf{0} & \mathbf{0} & \mathbf{0} \\
\mathbf{M}_{c_{1} p_{s}} & \mathbf{M}_{c_{1} c_{1}} & \mathbf{M}_{c_{1} p_{r}} & \mathbf{0} \\
\mathbf{0} & \mathbf{0} & \mathbf{M}_{p_{r} p_{r}} & \mathbf{0} \\
\mathbf{0} & \mathbf{0} & \mathbf{M}_{c_{2} p_{r}} & \mathbf{M}_{c_{2} c_{2}}
\end{array}\right], \\
\mathbf{D} & =\left[\begin{array}{cccc}
\mathbf{D}_{p_{s} p_{s}} & \mathbf{0} & \mathbf{D}_{p_{s} p_{r}} & \mathbf{0} \\
\mathbf{0} & \mathbf{D}_{c_{1} c_{1}} & \mathbf{0} & \mathbf{0} \\
\mathbf{D}_{p_{r} p_{s}} & \mathbf{0} & \mathbf{D}_{p_{r} p_{r}} & \mathbf{0} \\
\mathbf{0} & \mathbf{0} & \mathbf{0} & \mathbf{D}_{c_{2} c_{2}}
\end{array}\right], \\
\mathbf{K} & =\left[\begin{array}{cccc}
\mathbf{K}_{p_{s} p_{s}} & \mathbf{K}_{p_{s} c_{1}} & \mathbf{0} & \mathbf{0} \\
\mathbf{0} & \mathbf{K}_{c_{1} c_{1}} & \mathbf{0} & \mathbf{0} \\
\mathbf{0} & \mathbf{K}_{p_{r} c_{1}} & \mathbf{K}_{p_{r} p_{r}} & \mathbf{K}_{p_{r} c_{2}} \\
\mathbf{0} & \mathbf{0} & \mathbf{0} & \mathbf{K}_{c_{2} c_{2}}
\end{array}\right] .
\end{aligned}
$$

The vectors of the time functions $q_{i_{m_{x} m_{y}}}(t), g_{c_{n_{x n y} n_{z}}}(t)$ and $p_{t b l}(t)$ are defined as follows,

$$
\mathbf{y}(t)=\left[\begin{array}{l}
\mathbf{q}_{p_{s}}(t) \\
\mathbf{g}_{c_{1}}(t) \\
\mathbf{q}_{p_{r}}(t) \\
\mathbf{g}_{c_{2}}(t)
\end{array}\right], \quad \mathbf{x}(t)=\left[\begin{array}{c}
\mathbf{p}_{t b l}(t) \\
\mathbf{0} \\
\mathbf{0} \\
\mathbf{0}
\end{array}\right],
$$


in which the $\mathbf{p}_{t b l}(t)$ components are defined as follows,

$$
p_{t b l_{m_{x} m_{y}}}(t)=\int_{0}^{a} \int_{0}^{b} \psi_{p_{s m_{x} m_{y}}}(x, y) p_{t b l}(x, y, z, t) \mathrm{dxdy},
$$

where $\psi_{p_{s_{x} m_{y},}}(x, y)$ is the modal function for the source panel, see Eq. (12), and $p_{t b l}(x, y, z, t)$ is the external TBL excitation with $z=L_{c_{1}}$. The expressions for the matrices $\mathbf{M}, \mathbf{D}$ and $\mathbf{K}$ are given in Appendix A Additionally, by assuming $q_{i_{m_{x} m_{y}}}=Q_{i_{m_{x} m_{y}}} \mathrm{e}^{\mathrm{i} \omega t}$ and $g_{c_{n_{x} n_{y} n_{z}}}=G_{c_{n_{x} n_{y} n_{z}}} \mathrm{e}^{\mathrm{i} \omega t}$, the coupled structural acoustic system of Eq. [14] is expressed in the frequency domain, as [36]:

$$
\mathbf{Y}(\omega)=\mathbf{H}(\omega) \mathbf{X}(\omega),
$$

where

$$
\mathbf{Y}(\omega)=\left[\begin{array}{c}
\mathbf{Q}_{p_{s}}(\omega) \\
\mathbf{G}_{c_{1}}(\omega) \\
\mathbf{Q}_{p_{r}}(\omega) \\
\mathbf{G}_{c_{2}}(\omega)
\end{array}\right], \quad \mathbf{X}(\omega)=\left[\begin{array}{c}
\mathbf{P}_{t b l}(\omega) \\
\mathbf{0} \\
\mathbf{0} \\
\mathbf{0}
\end{array}\right],
$$

in which $\mathbf{Q}_{i}(\omega), \mathbf{G}_{c}(\omega)$ and $\mathbf{P}_{t b l}(\omega)$ are the frequency domain vectors of the displacements, pressure and turbulence pressure, respectively. The frequency response matrix of the system is given by $\mathbf{H}(\omega)$,

$$
\mathbf{H}(\omega)=\left[\begin{array}{cccc}
\mathbf{H}_{11}(\omega) & \mathbf{K}_{p_{s} c_{1}} & \mathrm{i} \omega \mathbf{D}_{p_{s} p_{r}} & \mathbf{0} \\
-\omega^{2} \mathbf{M}_{c_{1} p_{s}} & \mathbf{H}_{22}(\omega) & -\omega^{2} \mathbf{M}_{c_{1} p_{r}} & \mathbf{0} \\
\mathrm{i} \omega \mathbf{D}_{p_{r} p_{s}} & \mathbf{K}_{p_{r} c_{1}} & \mathbf{H}_{33}(\omega) & \mathbf{K}_{p_{r} c_{2}} \\
\mathbf{0} & \mathbf{0} & -\omega^{2} \mathbf{M}_{c_{2} p_{r}} & \mathbf{H}_{44}(\omega)
\end{array}\right]^{-1}
$$

with

$$
\begin{aligned}
& \mathbf{H}_{11}(\omega)=-\omega^{2} \mathbf{M}_{p_{s} p_{s}}+\mathrm{i} \omega \mathbf{D}_{p_{s} p_{s}}+\mathbf{K}_{p_{s} p_{s}}, \\
& \mathbf{H}_{22}(\omega)=-\omega^{2} \mathbf{M}_{c_{1} c_{1}}+\mathrm{i} \omega \mathbf{D}_{c_{1} c_{1}}+\mathbf{K}_{c_{1} c_{1}}, \\
& \mathbf{H}_{33}(\omega)=-\omega^{2} \mathbf{M}_{p_{r} p_{r}}+\mathrm{i} \omega \mathbf{D}_{p_{r} p_{r}}+\mathbf{K}_{p_{r} p_{r}}, \\
& \mathbf{H}_{44}(\omega)=-\omega^{2} \mathbf{M}_{c_{2} c_{2}}+\mathrm{i} \omega \mathbf{D}_{c_{2} c_{2}}+\mathbf{K}_{c_{2} c_{2}} .
\end{aligned}
$$

The contributions of the passive damping force and the active damping force, $\tilde{f}_{p}$ and $\tilde{f}_{c}$, are included in $\mathbf{H}(\omega)$ as follows,

$$
\begin{aligned}
& \mathbf{D}_{p_{s} p_{s}}=\operatorname{diag}\left[2 \rho t \omega_{m} \sigma\right]_{p_{s}}+c_{p} \boldsymbol{\psi}_{p_{s}}^{2}, \\
& \mathbf{D}_{p_{r} p_{s}}=-c_{p} \boldsymbol{\psi}_{p_{s}} \boldsymbol{\psi}_{p_{r}}, \\
& \mathbf{D}_{p_{s} p_{r}}=-\left(g+c_{p}\right) \boldsymbol{\psi}_{p_{s}} \boldsymbol{\psi}_{p_{r}}, \\
& \mathbf{D}_{p_{r} p_{r}}=\operatorname{diag}\left[2 \rho t \omega_{m} \sigma\right]_{p_{r}}+\left(g+c_{p}\right) \boldsymbol{\psi}_{p_{r}}^{2},
\end{aligned}
$$

where $\omega_{m}$ are the natural frequencies of the panels. In Eq. 22, $\psi_{i}$ are the vectors of the modal functions $\psi_{i_{m_{x} m y}}(x, y)$ at the location of the active vibration isolation unit. 
Since the TBL excitation is defined in terms of the power spectral density (PSD) of the wall pressure, it is convenient to write the coupled system governing equations in the PSD domain,

$$
\mathbf{S}_{Y Y}(\omega)=\mathbf{H}^{*}(\omega) \mathbf{S}_{X X}(\omega) \mathbf{H}^{\mathbf{T}}(\omega)
$$

where $\mathbf{S}_{X X}(\omega)$ is the PSD matrix of the random excitation, which includes the PSD matrix of the TBL pressure; $\mathbf{S}_{Y Y}(\omega)$ is the PSD matrix of the plate displacements, $\mathbf{S}_{W W_{i}}(\omega)$, and the acoustic pressures, $\mathbf{S}_{P P_{c}}(\omega)$, and the superscripts $*$ and T denote Hermitian and matrix transpose, respectively [36]. The PSD matrix of the TBL pressure is given by $\mathbf{S}_{t b l}(\omega)$, with components defined as follows,

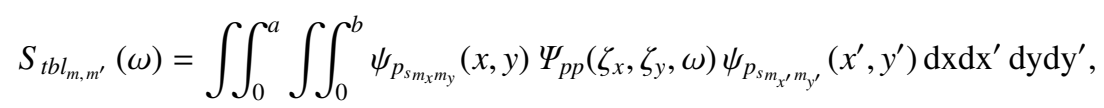

in which $\psi_{p_{s_{x} m_{y}}}(x, y)$ is the modal function for the source panel, see Eq. (12), and $\Psi_{p p}\left(\zeta_{x}, \zeta_{y}, \omega\right)$ is the Corcos CPSD function, see Eq. (1)-(2). For notation simplicity, in Eq. (24) the indexes $m$ and $m^{\prime}$ are used to indicate the set $\left(m_{x}, m_{y}\right)$ and $\left(m_{x^{\prime}}, m_{y^{\prime}}\right)$, respectively. Eq. (24) gives the coupling between the pressure field and the mode shapes of the structure. In the following study, the influence of the boundary layer wavenumber-frequency spectrum on the structural response is defined by fixing the structure dimensions and the external flow properties, as done in Sec. 3 After some mathematical manipulations, the PSD functions of the plate displacement (and velocity) and the acoustic enclosure pressure in a specific point inside the enclosure can be defined,

$$
\begin{aligned}
& \mathbf{S}_{w w i}\left(x_{1}, y_{1}, x_{2}, y_{2}, \omega\right)=\sum_{m_{x_{1}}, m_{x_{2}}}^{M_{x_{i}}^{2}} \sum_{m_{y_{1}}, m_{y_{2}}}^{M_{y_{i}}^{2}} \psi_{i_{m_{x_{1}} m_{y_{1}}}}\left(x_{1}, y_{1}\right) S_{W W i_{m_{1}, m_{2}}}(\omega) \psi_{i_{m_{x_{2}} m_{y_{2}}}}\left(x_{2}, y_{2}\right), \\
& \mathbf{S}_{p p_{c}}\left(x_{1}, y_{1}, z_{1}, x_{2}, y_{2}, z_{2}, \omega\right)= \sum_{n_{x_{1}}, n_{x_{2}}}^{N_{x_{c}}^{2}} \sum_{n_{y_{1}}, n_{y_{2}}}^{N_{y_{c}}^{2}} \sum_{n_{z_{1}}, n_{z_{2}}}^{N_{z_{c}}^{2}} \Gamma_{c_{n_{x_{1}} n_{y_{1}} n_{z_{1}}}}\left(x_{1}, y_{1}, z_{1}\right) \\
& \times S_{P P c_{n_{1}, n_{2}}}(\omega) \Gamma_{c_{n_{x_{2}} n_{y_{2}} n_{z_{2}}}}\left(x_{2}, y_{2}, z_{2}\right),
\end{aligned}
$$

in which $S_{W W i_{m_{1}, m_{2}}}(\omega)$ and $S_{P P c_{n_{1}, n_{2}}}(\omega)$ are the $\mathbf{S}_{W W_{i}}(\omega)$ and $\mathbf{S}_{P P_{c}}(\omega)$ matrix components, respectively. Note that the indexes $m$ and $n$ are used to indicate $\left(m_{x}, m_{y}\right)$ and $\left(n_{x}, n_{y}, n_{z}\right)$, respectively, and, $m_{1}, m_{2}, n_{1}, n_{2}$ are referred to the specific point $\left(x_{1}, y_{1}, z_{1}\right)$ and $\left(x_{2}, y_{2}, z_{2}\right)$, respectively.

The radiating panel velocity PSD is given by

$$
\mathbf{S}_{v v_{i}}\left(x_{1}, y_{1}, x_{2}, y_{2}, \omega\right)=\omega^{2} \mathbf{S}_{w w_{i}}\left(x_{1}, y_{1}, x_{2}, y_{2}, \omega\right) .
$$

\section{Results with a reduced order model}

\subsection{Description of the simplified model}

In this section, the stability and performance analysis of the active control system are carried out using a simplified model. The simplified model is obtained by considering only the lowest order uncoupled modes for each panel $(1,1)$ and for each air cavity $(0,0,0)$.

Note that the first mode of each air cavity is characterised by a uniform pressure variation throughout the cavity volume and by a zero natural frequency. The resulting coupled system 


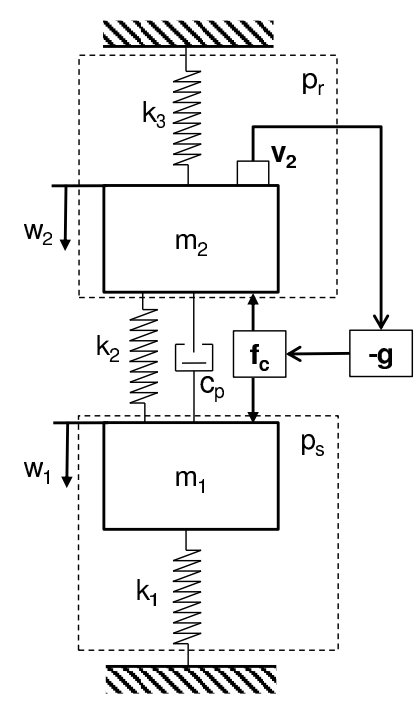

Figure 2: Reduced order model.

then behaves exactly as a two degree of freedom (DOF) mechanical system which, as shown in Fig. 2, is represented through mass and stiffness parameters $m_{1}, m_{2}, k_{1}, k_{2}, k_{3}$, and which is characterised by two coupled mode shapes and two natural frequencies. By using the two mode assumption, the governing equations are defined as in Eq. (14)-(22), in which $\mathbf{M}, \mathbf{K}$ and $\mathbf{D}$ are 4-by-4 matrices. Under such assumption the masses and stiffnesses of an equivalent lumped parameter model, schematically shown in Fig. 2 can be directly calculated using Eq. (18) and are given in Appendix B Note that the $(x, y)$ locations of the excitation force, the sensor and the actuator, are absorbed in the expressions for the equivalent stiffnesses $k_{1}, k_{2}$ and $k_{3}$. Considering the fact that the pressures inside the two cavities are uniform in this approximation, the stiffness of the air enclosed in $c_{1}$ can be represented by the stiffness $k_{2}$, and the stiffness of the air enclosed in the cavity $c_{2}$ contributes to the stiffness $k_{3}$. In the simplified model the damping ratios $\sigma$ and $\sigma_{a c}$ are neglected, such that the only damping in the system is achieved through the feedback gain $g$ and the damper $c_{p}$. This enables a significantly easier derivation of the stability and performance metrics presented later in the text. The remaining geometrical and physical properties used throughout the paper are shown in Table 11. These are chosen to match the properties of a set of panels used in an ongoing experimental study where the panels are printed circuit boards with embedded wiring to five sensor-actuator units. If a point force excitation is assumed on the mass $m_{1}$, the frequency response function between the velocity of the radiating body, i.e. $p_{r}$, and the excitation force is as follows,

$$
\begin{array}{r}
y_{p_{r}, f}(\mathrm{i} \omega)=\frac{-\omega^{2} c_{p}+\mathrm{i} \omega k_{2}}{m_{1} m_{2} \omega^{4}-\mathrm{i} \omega^{3}\left(\left(m_{2}+m_{1}\right) c_{p}+m_{1} g\right)-\omega^{2}\left(\left(m_{2}+m_{1}\right) k_{2}+m_{2} k_{1}+k_{3} m_{1}\right)+} \\
+\mathrm{i} \omega\left(\left(k_{1}+k_{3}\right) c_{p}+k_{1} g\right)+\left(k_{2}+k_{3}\right) k_{1}+k_{2} k_{3} .
\end{array}
$$


The pressure in the acoustic cavity $c_{2}$ due to the excitation force is directly related to the displacement of $m_{2}$ as follows,

$$
p_{c_{2}, f}(\mathrm{i} \omega)=C_{0} \frac{y_{p_{r}, f}(\mathrm{i} \omega)}{\mathrm{i} \omega},
$$

with

$$
C_{0}=\frac{-4 \rho_{0} c_{0}^{2}}{\pi^{2} L_{c_{2 z}}},
$$

where $c_{0}$ and $\rho_{0}$ are the speed of sound and density of the air in the two acoustic enclosures. The pressure in the cavity $c_{1}$ is proportional to the relative displacement of the two bodies and it is given by,

$$
p_{c_{l}, f}(\mathrm{i} \omega)=\frac{C_{1}}{\mathrm{i} \omega}\left(y_{p_{r} f}(\mathrm{i} \omega)-y_{p_{s} f}(\mathrm{i} \omega)\right),
$$

with

$$
C_{1}=\frac{4 \rho_{0} c_{0}^{2}}{\pi^{2} L_{c_{1 z}}},
$$

\begin{tabular}{lr}
\hline Properties of the external flow & \\
\hline \hline Free stream velocity $U_{\infty}\left(\mathrm{m} \mathrm{s}^{-1}\right)$ & 240 \\
Boundary layer thickness $\delta(\mathrm{m})$ & 0.08 \\
Kinematic viscosity $v$ & $3.3553 \times 10^{-5}$ \\
Friction velocity $U_{\tau}\left(\mathrm{m} \mathrm{s}^{-1}\right)$ & 6.75 \\
External sound speed $c\left(\mathrm{~m} \mathrm{~s}^{-1}\right)$ & 300 \\
External air density $\rho\left(\mathrm{kg} \mathrm{m}^{-3}\right)$ & 0.44 \\
Empirical parameters $\alpha_{x} / \alpha_{y}$ & $0.10 / 0.77$ \\
\hline \hline Properties of the acoustic enclosures $\left(c_{1}\right.$ and $\left.c_{2}\right)$ & 343 \\
\hline \hline Internal sound speed $c_{0}\left(\mathrm{~m} \mathrm{~s}^{-1}\right)$ & 1.19 \\
Internal air density $\rho_{0}\left(\mathrm{~kg} \mathrm{~m}^{-3}\right)$ & 0.4 \\
Length $L_{c_{x}}(\mathrm{~m})$ & 0.3 \\
Width $L_{c_{y}}(\mathrm{~m})$ & 0.038 \\
Height $L_{c_{1}}(\mathrm{~m})$ & 0.4 \\
Height $L_{c_{2}}(\mathrm{~m})$ & 0.05 \\
Damping ratio $\sigma_{a c}$ & 0.4 \\
\hline \hline Properties of the panels $\left(p_{r}\right.$ and $\left.p_{s}\right)$ & 0.3 \\
\hline \hline Length $a(\mathrm{~m})$ & 100 \\
Width $b(\mathrm{~m})$ & 2380 \\
Elasticity Modulus $E(\mathrm{G} \mathrm{Pa})$ & 0.33 \\
Density $\rho(\mathrm{kg}$ m & \\
Poisson's ratio $v$ & $0.002(0.003)$ \\
Source plate thickness $t_{p_{s}}(\mathrm{~m})$ & 0.01 \\
Radiating plate thickness $t_{p_{r}}(\mathrm{~m})$ & \\
Structural damping ratio $\sigma$ & $0.002)$ \\
\hline
\end{tabular}

Table 1: Model properties. 
and $y_{p_{s} f}(\mathrm{i} \omega)$ is given by:

$$
\begin{array}{r}
y_{p_{s} f}(\mathrm{i} \omega)=\frac{\mathrm{i} \omega\left(-m_{2} \omega^{2}+\mathrm{i}\left(g+c_{p}\right) \omega+k_{2}+k_{3}\right)}{m_{1} m_{2} \omega^{4}-\mathrm{i} \omega^{3}\left(\left(m_{2}+m_{1}\right) c_{p}+m_{1} g\right)-\omega^{2}\left(\left(m_{2}+m_{1}\right) k_{2}+m_{2} k_{1}+k_{3} m_{1}\right)+} \\
+\mathrm{i} \omega\left(\left(k_{1}+k_{3}\right) c_{p}+k_{1} g\right)+\left(k_{2}+k_{3}\right) k_{1}+k_{2} k_{3} .
\end{array}
$$

At this point the problem is made dimensionless in order to simplify and generalise the analysis. To this end, the following non-dimensional parameters are used:

$$
\begin{aligned}
\mu & =\frac{m_{2}}{m_{1}}, \\
\alpha & =\left(\frac{\Omega_{2}}{\Omega_{1}}\right)^{2}, \\
\beta & =\left(\frac{\Omega_{3}}{\Omega_{1}}\right)^{2}, \\
\eta & =\frac{c_{p}}{2 \sqrt{k_{1} m_{1}}}, \\
\xi & =\frac{g}{c_{p}},
\end{aligned}
$$

where $\Omega_{1}=\sqrt{k_{1} / m_{1}}, \Omega_{2}=\sqrt{k_{2} / m_{2}}, \Omega_{3}=\sqrt{k_{3} / m_{2}}, \mu$ is the mass ratio, $\alpha$ and $\beta$ are the frequency ratios and $\xi$ and $\eta$ are the active and passive damping ratios, respectively. Using the non-dimensional frequency $\Omega=\omega / \Omega_{1}$, substituting Eq. [34e) into Eq. (28) and then normalising by $\Omega_{1} m_{1}$, the dimensionless frequency response function between the velocity of the radiating panel and the force on the source panel can be expressed as follows,

$$
y_{p_{r} f}(\mathrm{i} \Omega)=\frac{-\Omega^{2} B_{2}+\mathrm{i} \Omega B_{1}}{A_{4} \Omega^{4}-A_{3} \mathrm{i} \Omega^{3}-A_{2} \Omega^{2}+A_{1} \mathrm{i} \Omega+A_{0}},
$$

where the coefficients are given by:

$$
\begin{aligned}
& B_{1}=\alpha \mu, \\
& B_{2}=2 \eta, \\
& A_{0}=\mu(\alpha \beta \mu+\alpha+\beta), \\
& A_{1}=2 \eta(\beta \mu+\xi+1), \\
& A_{2}=\mu(\alpha \mu+\alpha+\beta+1), \\
& A_{3}=2 \eta(\xi+\mu+1), \\
& A_{4}=\mu .
\end{aligned}
$$

At the first natural frequency the two panels move in phase and at the second natural frequency they move out of phase. Both modes are characterised by large volumetric vibration components (i.e. large volume velocity), so that large acoustic pressure fluctuations in the back cavity associated with the two modes can be expected, as discussed later in the text and as shown in Fig. D.21-a and b in Appendix D It is reasonable to place the sensor-actuator unit near the centre of the panels in the $(x, y)$ plane since in such a case the sensor/actuator can couple well to the 
vibration governed by the two modes.

By substituting $s=\mathrm{i} \Omega$ into the denominator of Eq. (35) the dimensionless characteristic equation of the reduced order model shown in Fig. 2] is obtained:

$$
A_{4} s^{4}+A_{3} s^{3}+A_{2} s^{2}+A_{1} s+A_{0}=0,
$$

where $s$ is the dimensionless Laplace variable. In the following subsection the stability of the active control system is examined using the characteristic equation Eq. (37).

\subsection{Stability}

A system is stable if all roots of its characteristic equation have negative real parts. The Routh-Hurwitz criterion can be used to verify this without finding the roots of a possibly high order polynomial. According to this criterion, the necessary condition for the stability is that all coefficients of the characteristic equation are positive. Additionally, all the principal diagonal minors $\Delta_{i}$ of the Hurwitz matrix must be positive.

The principal diagonal minors $\Delta_{i}$ of the Hurwitz matrix of the system at hand can be calculated as follows,

$$
\begin{gathered}
\Delta_{1}=A_{1}=2 \eta(\beta \mu+\xi+1), \\
\Delta_{2}=A_{3}=2 \eta(\xi+\mu+1), \\
\Delta_{3}=\left|\begin{array}{cc}
A_{3} & A_{4} \\
A_{1} & A_{2}
\end{array}\right|=2 \xi \mu \eta((\mu+1) \alpha+\beta)+2 \mu \eta\left((\mu+1)^{2} \alpha+\mu+\beta\right), \\
\Delta_{4}=\left|\begin{array}{ccc}
A_{3} & A_{4} & 0 \\
A_{1} & A_{2} & A_{3} \\
0 & A_{0} & A_{1}
\end{array}\right|=4(1-\beta) \mu^{2} \eta^{2}\left(\xi^{2} \alpha+\xi(1-\beta+(\mu+1) \alpha)+1-\beta\right) .
\end{gathered}
$$

Then, the conditions that must be satisfied simultaneously are the following:

$$
\left(\Delta_{1}>0\right) \wedge\left(\Delta_{2}>0\right) \wedge\left(\Delta_{3}>0\right) \wedge\left(\Delta_{4}>0\right) .
$$

This results in a system of inequalities, which can be solved for the active damping ratio $\xi$. In Fig. 3] the four principal diagonal minors, Eq. (38)-(41), are plotted as function of $\xi$ for two cases: (a) $\beta>1$; (b) $\beta<1$. It can be seen in Fig. 3 that there are three principal diagonal minors with linear dependence on the active damping ratio, and one with a quadratic dependence. It can also be seen that the stability substantially depends on the frequency ratio $\beta$, whose variations can be accomplished by, for example, changing the relative thicknesses of the two panels. In fact, in both cases it can be shown that it is the fourth principal diagonal minor $\Delta_{4}$ with the quadratic dependence on $\xi$, that governs the system stability.

In case (a) $\beta>1$ the radiating panel fundamental natural frequency, $\Omega_{3}$, is larger than the source panel fundamental natural frequency, $\Omega_{1}$, and in case (b) $\beta<1$ the situation is opposite. In case $\beta>1$, it can be calculated using Eq. (41) that the system is stable if the active damping ratio is between two bordering values given by

$$
\xi_{1,2}=-\frac{\alpha(1+\mu)+1-\beta \mp \sqrt{(1+\mu)^{2} \alpha^{2}-2 \alpha(\beta-1)(\mu-1)+(\beta-1)^{2}}}{13^{2 \alpha}} .
$$


This is because, as shown in Fig. 3-a, all principal diagonal minors $\Delta_{i}$ are positive if $\xi_{1}>\xi>\xi_{2}$ due to the fact that the quadratic coefficient in the fourth principal diagonal minor is negative (the parabola is oriented downwards in Fig. 3.a). On the contrary, if $\beta<1$ then the system is stable for any $\xi>\xi_{1}$, as shown in Fig. 3.b where the parabola is oriented upwards. In conclusion, the range of stable feedback gains is found as follows,

$$
\begin{gathered}
\forall \beta>1, \quad \xi_{1}>\xi>\xi_{2}, \\
\forall \beta<1, \quad \xi>\xi_{1} .
\end{gathered}
$$

It can be stated that in the case with $\beta<1$ the source body, having the larger uncoupled natural frequency than the radiating body, behaves more like a fixed reference base against which the actuator can react without pronounced feedthrough effect that could otherwise compromise stability properties. This is related to the fact that a successful implementation of a direct feedback control on a large flexible structure (without a model of the sensor-actuator frequency response function), requires that the sensor and actuator form a dual and collocated pair [38]. Dual sensoractuator pairs are those which complement each other in terms of power (i.e. velocity sensor and a force actuator, or a moment actuator and an angular velocity sensor). However, in the present study, the actuator generates the force on the radiating panel while reacting against the source panel. The action force component is indeed collocated to the velocity sensor mounted on the radiating panel. On the other hand, the reaction force component has no dedicated collocated sensor on the source panel. The frequency response function between the reaction force component and the velocity sensor is thus responsible for the stability problems in case $\beta>1$. This is because frequency response functions between two different points of a flexible structure do not necessarily have their phases bound within a 180 degree range, whereas the driving point FRFs do have their phase limited within a 180 degrees range. In particular, driving point mobilities have their phase limited in the range between +90 and -90 degrees. The sensor-actuator open loop frequency response functions $(\mathrm{OL}-\mathrm{FRF})$ can therefore be used through Nyquist stability criterion, as discussed in Section 4

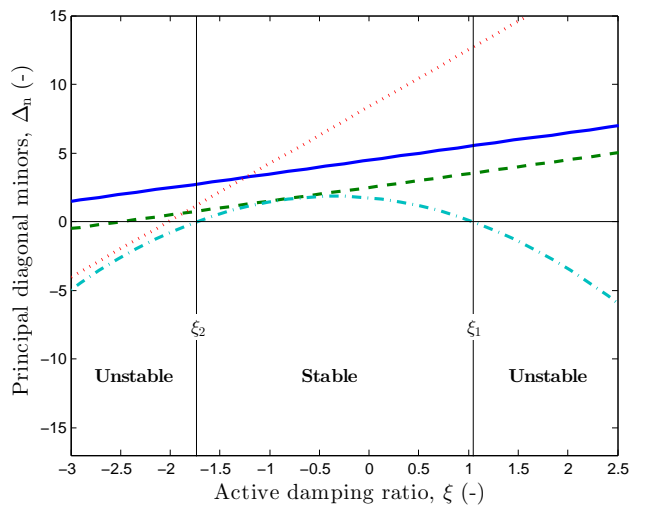

(a)

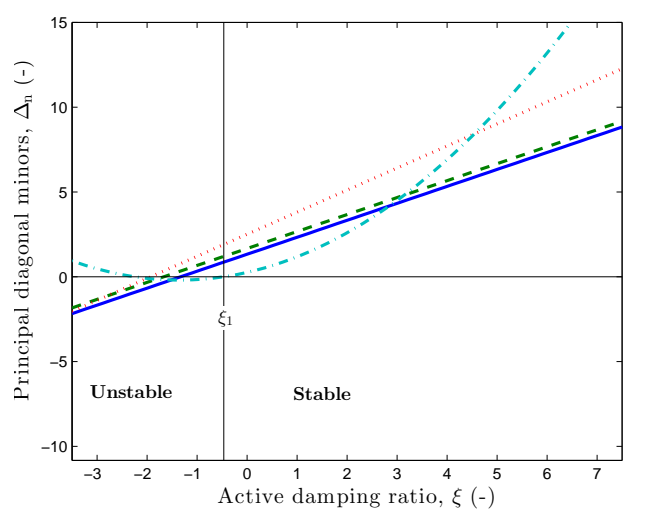

(b)

Figure 3: Principal diagonal minors plotted versus the active damping ratio $\xi,(-) \Delta_{1},(--) \Delta_{2},(\cdots) \Delta_{3},(-\cdot-) \Delta_{4}: \beta>1$ (a); $\beta<1$ (b). 


\subsection{Performance}

In this section, the performance of the active control system is analysed by using two different load conditions. Firstly, a white noise forcing is assumed on $m_{1}$, Fig. 2, where the mass $m_{1}$ represents the source panel (see Appendix B) and is thus referred to as the source body. Then, the mean squared velocity of the mass $m_{2}$ is found by calculating the integral over all frequencies of the squared magnitude of the transfer mobility, Eq. (28), of the system in Fig. 2. The mass $m_{2}$ in this case represents the radiating panel and is referred to as the radiating body. Note that the mean squared displacement of the mass $m_{2}$ is proportional to the mean squared pressure in the cavity $c_{2}$ according to the two mode assumption, see Eq. (29). Thus, in the two mode approximation, the problem of vibration transmission in the mechanical system shown in Fig. 2 is fully representative of the problem of sound transmission into the cavity $c_{2}$. Secondly, a TBL excitation on the source panel is assumed. In this case, the frequency- and space-averaged velocity PSD of the radiating panel is used. In either excitation scenario, the performance of the active control is studied as a function of the active and passive damping ratio $\xi$ and $\eta$.

\subsubsection{Point force excitation}

In the case of the excitation force having a uniform PSD equal to unity, the mean squared velocity of the radiating body is given by the following integral:

$$
I_{p_{r}, f}=\int_{-\infty}^{+\infty}\left|y_{p_{r},}(\mathrm{i} \Omega)\right|^{2} \mathrm{~d} \Omega
$$

where $\left|y_{p_{r} .}(\mathrm{i} \Omega)\right|^{2}$ is the squared magnitude of the system transfer mobility, that is given by Eq. 35. The integral can be calculated using formulae given in [39] leading to,

$$
I_{p_{r}, f}=\frac{-2 \pi\left(\left(1 / 4 \alpha^{2} \mu^{2}+\eta^{2}\right) \xi+1 / 4 \mu^{2}(\mu+1) \alpha^{2}+\eta^{2}(\beta \mu+1)\right)}{\eta \mu^{2}(\beta-1)\left(\alpha \xi^{2}+((\mu+1) \alpha-\beta+1) \xi-\beta+1\right)} .
$$

The mean squared velocity of the radiating body is plotted as function of the passive and active damping ratio in Fig. 4 The case with $\beta>1$ is shown in Fig. 4 - and ane with $\beta<1$ is shown in Fig. 4.b, following a similar layout as in Fig. 3. It can be seen that for $\beta>1$, a minimum exists for a certain combination of $\xi$ and $\eta$ which is marked by a white asterisk “*”in Fig. 4-a. Thus there is an optimal combination of the active and passive damping ratios, $\left(\xi_{\text {opt }}, \eta_{\text {opt }}\right)$, when $\beta>1$. On the other side, for $\beta<1$, there is no minimum since the kinetic energy decreases monotonically with increasing the active damping ratio $\xi$. This is however only due to a) the limitations of the reduced order model and its inability to capture the high-frequency behaviour of the double panel structure, and b) the fact that idealised sensor and actuator transducers are assumed. Therefore, the active damping ratios $\xi$, corresponding to the optimal passive damping ratio $\eta_{o p t}$, is set to 10 and it is marked by the white asterisk “*”in Fig. 4 b, in order to qualitatively illustrate the performance of the active control. Therefore, when $\beta<1$, large absolute values of positive $\xi$ (large feedback gain) give considerable radiating body vibration reduction effects which result in considerable sound transmission reduction effects. On the contrary, when $\beta>1$, the maximum dimensionless gain is limited between $\xi_{1}$ and $\xi_{2}$. In both figures, the white dashdotted curves give the optimal passive damping ratio, $\eta_{\text {opt }}$, for any stable $\xi$. The equation of the curve can be calculated by differentiating by $\eta$ the right hand side of Eq. (47) and solving $\frac{\partial I_{p_{r}, f}}{\partial \eta}=0$ for $\eta$ which yields,

$$
\eta_{o p t}=\frac{\mu \alpha}{2} \sqrt{\frac{\mu+1+\xi}{\beta \mu+1+\xi}}
$$


For $\xi=0$ the optimal passive damping ratio, $\eta_{\text {opt }}(\xi=0)$, is obtained from Eq. (48) and it is indicated by a white circle for both cases in Fig. 4 . If the active damping ratio $\xi$ is a large number, which is relevant for systems of type (b) with $\beta<1$, then the optimal passive damping ratio becomes independent of the active damping ratio and can be approximated by:

$$
\eta_{o p t_{\xi \gg}} \approx \lim _{\xi \rightarrow+\infty} \eta_{o p t}=\frac{\alpha \mu}{2}
$$

In Fig. 5. the non-dimensional mobility $y_{p_{s} .}$, Eq. (35), for the two cases of $\beta$ is shown as a function of normalized frequency $\Omega=\omega / \Omega_{1}$. Two control approaches are considered. The first approach is a fully passive approach where the feedback gain equals zero $(\xi=0)$ and the damping is achieved exclusively through the optimal passive damping ratio $\eta$, indicated by the circle in Fig. 4. The second approach is with a combination of passive and active damping achieved through both the control actuator and the damper $c_{p}$. In this case, the optimally tuned damping ratio pair, $\left(\xi_{o p t}, \eta_{o p t}\right)$, is used when $\beta>1$, and, for $\beta<1, \xi$ is set to 10 as indicated in Fig. 4 with the white asterisk “*”. The passive damping ratio is again set to the optimal value that lies on the white dash-dotted line in Fig. 4. Comparing the frequency spectra in Fig. 5, it can be seen that the active control (solid line) gives an improvement over the passive one in either case of $\beta$. However, the situation in which $\beta<1$ results in a much more convincing active reductions of the radiating panel vibrations in comparison to the situation in which $\beta>1$.

This is because in this case the active control approach where $\beta<1$ can avoid the limitations of the passive approach entirely, since the velocity spectra of the radiating body decrease in a broad frequency band as the feedback gain is increased, see Fig. 5-b. Therefore, the frequency-averaged vibrations of the radiating panel reduce along the dash-dotted white line in Fig. 4 -b. With $\beta>1$ the performance is not as promising, as shown in Fig. 4 - a and Fig. 5 - a. The active control can still outperform the passive control, as can be seen by comparing for example the contour levels at the asterisk (optimal active control) and the circle (optimal passive control) in Fig. 4-a. However the broadband performance improvement is only about one $\mathrm{dB}$. This is because the active

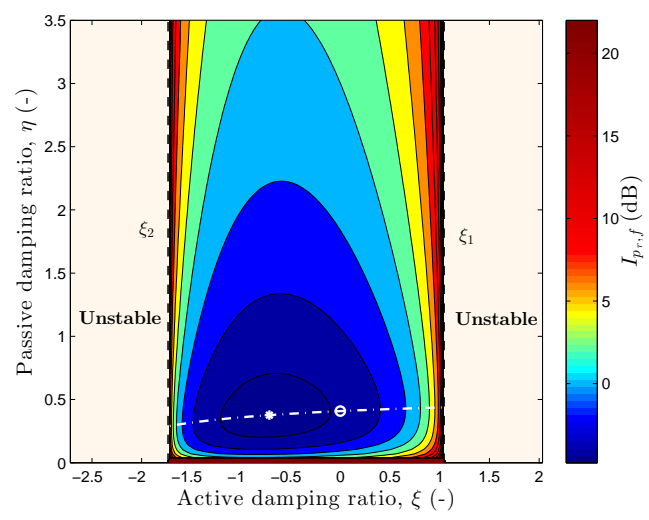

(a)

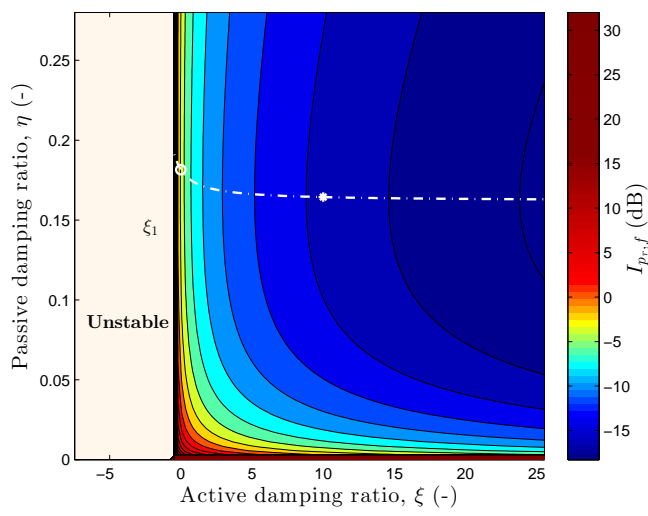

(b)

Figure 4: Mean squared velocity of the radiating body, in the non-dimensional form, Eq. 47, plotted versus $\xi$ and $\eta$ in case of a white noise excitation, with $\eta_{o p t}(\xi=0)$ (white o): $\beta>1$ with $\left(\xi_{\text {opt }}, \eta_{\text {opt }}\right)$ (white $\left.*\right)$ (a); $\beta<1$ with $(\xi=10$, $\left.\eta_{o p t}(\xi=10)\right)($ white $*)(\mathrm{b})$. 
damping ratio (and thus the feedback gain) is limited between the two values $\xi_{1}$ and $\xi_{2}$ as shown in Fig. 3.-a. Therefore, the reductions of the sound transmission at the low frequency resonances due to the added active damping is also limited. In addition, the roll-off at higher dimensionless frequencies, above approximately 2.1, is compromised as shown in Fig. 5.a.

Then, it should be noted that the optimum combination of the passive and active damping ratio in case $\beta>1$ can, in certain cases, such as is the case shown in Fig. 4-a, require a negative active damping ratio, which corresponds to the active "undamping" or positive velocity feedback. The underlying reason for this is that in such situations, where the stability of the system is compromised, it would be better to place the sensor on the source panel and implement a direct source panel velocity feedback [24].

It is thus reasonable to consider passive control for pairs of panels characterised by $\beta>1$. In such a case the mean squared velocity of the radiating body in the non-dimensional form is given by

$$
I_{p_{r}, f}^{P}=\frac{2 \pi\left(1 / 4 \mu^{2} \alpha^{2}(\mu+1)+\eta^{2}(\beta \mu+1)\right)}{\eta \mu^{2}(\beta-1)^{2}} .
$$

Eq. 50 can be obtained by substituting $\xi=0$ in Eq. 477). The variation of $I_{p_{r}, f}^{P}$ with the damping ratio $\eta$ is shown in Fig. 6-a for the case with $\beta>1$. The optimum choice of the damping ratio is found to be of about 0.42 for the example system at hand. It can be calculated by minimising the right hand side of Eq. 50 and $\frac{\partial I_{r, f}^{P}}{\partial \eta}=0$, which yields:

$$
\eta_{o p t}^{P}=\frac{\mu \alpha}{2} \sqrt{\frac{\mu+1}{\beta \mu+1}} .
$$

This value is indicated by the hollow circle in Fig. 4

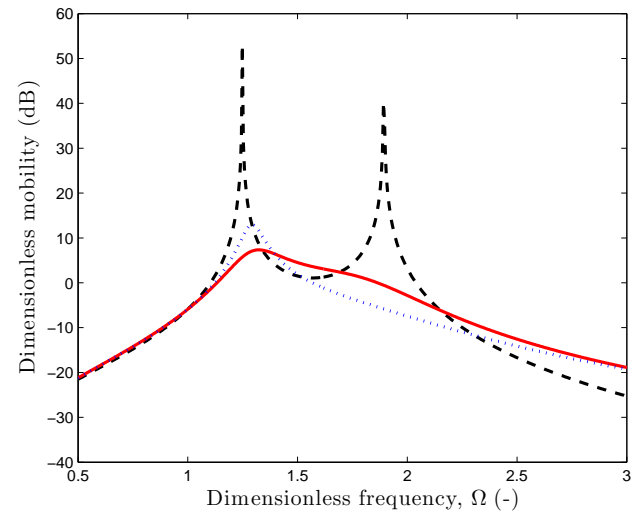

(a)

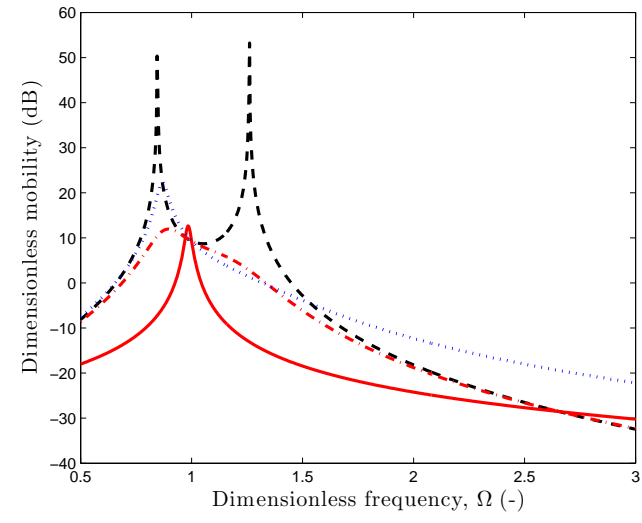

(b)

Figure 5: Amplitude of the dimensionless transfer mobility of the radiating body, Eq. 35: $\beta>1$ (a); $\beta<1$ (b). No control $(--)$, passive control with $\eta_{\text {opt }}(\xi=0)(\cdots)$, optimal tuned active control with $\left(\xi_{\text {opt }}, \eta_{\text {opt }}\right)$ for $\beta>1$ and with $\left(\xi=10, \eta_{\text {opt }}(\xi=10)\right)(-)$ and $\left(\xi=10, \eta=\eta_{\text {opt }} / 10\right)(-\cdot-)$ for $\beta<1$. 


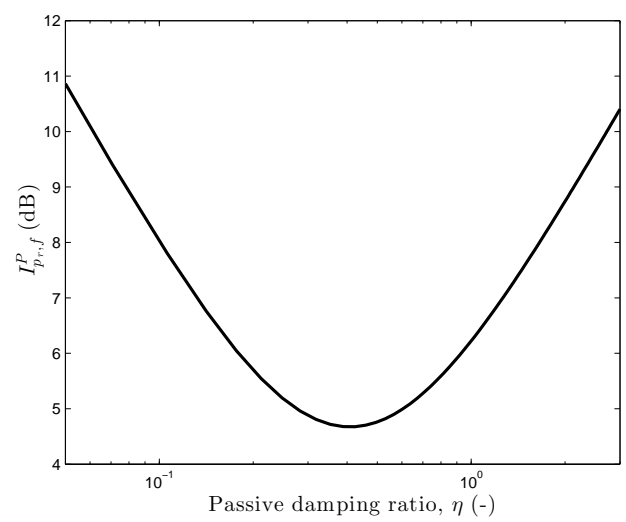

(a)

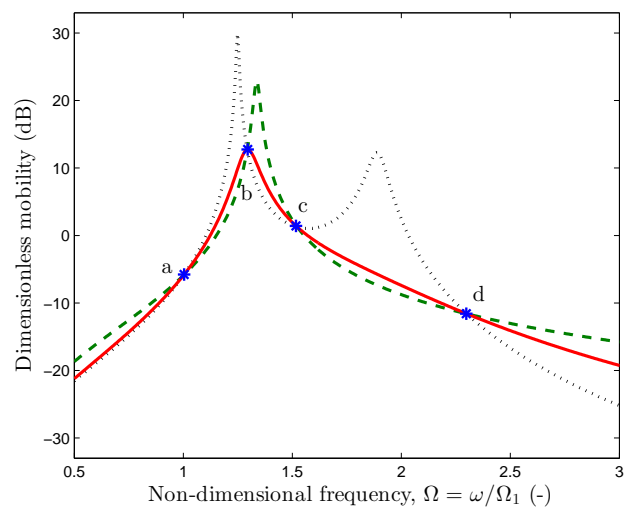

(b)

Figure 6: Dimensionless isolation performance index, $I_{p_{r}, f}^{P}$ of Eq. 50 plotted versus the damping ratio $\eta$, case with $\beta>1$ (a). Amplitude of the dimensionless transfer mobility $\left|y_{p_{r}, f}^{P}(\mathrm{i} \Omega)\right|$ (see Eq. 35 ) plotted versus $\Omega$ for $(\cdots)$ low damping, (--) high damping and (-) optimal damping, case with $\beta>1$ (b).

The physical reasons for the existence of this optimum are discussed next. In Fig. 6-b, the amplitude of the dimensionless transfer mobility is plotted versus $\Omega$ for three different damping coefficients. The black dotted line is for a small damping ratio, 0.03 , the red solid line is for the optimal damping ratio and the green dashed line is for a large damping ratio 2.4.

It can be seen in Fig. 6-b that if the damping ratio is small, then the vibrations of the radiating panel are very large at the two resonances, and if the damping ratio is large, then a new resonance appears approximately at $\Omega=\Omega_{1} \sqrt{\frac{1+\beta \mu}{1+\mu}}$, which in this case is about 1.35 . This is because the very large damping coefficient virtually cancels the relative radiating/source panels' velocity, and the two panels become connected through a locked damper. This has consequences to the high frequency vibration and sound radiation which in this case becomes closer to that of a single panel, i.e. the benefits of the high-frequency roll-off characteristic for double panels are lost. Also, there are four frequencies at which the vibration of the radiating panel is independent on damping ratio $\eta$, which are indicated in Fig. 6 -b by the blue dots. These points separate five frequency regions. At frequencies around the resonances, between $\Omega_{a}$ and $\Omega_{b}$, and between $\Omega_{c}$ and $\Omega_{d}$, the increase in the damping coefficient decreases the vibration transmissibility. Otherwise, at frequencies below $\Omega_{a}$, between $\Omega_{b}$ and $\Omega_{c}$, and above $\Omega_{d}$ the increase in the damping coefficient increases the vibration transmissibility. These frequencies are calculated and given in Appendix C. In conclusion, the inconsistent impact of the passive damping ratio at various frequency ranges is the reason why the frequency averaged kinetic energy of the radiating panel plotted in Fig. 6- a has a minimum which corresponds to the optimal passive damping coefficient $\eta_{\text {opt }}$.

Tuning the damper coefficient to the optimal value, by substituting Eq. (51) in Eq. (50), the 
integral kinetic energy index becomes:

$$
I_{p_{r}, f_{o p t}}^{P}=\frac{2 \alpha \pi \sqrt{(\mu+1)(\beta \mu+1)}}{\mu(\beta-1)^{2}} .
$$

Eq. (52) suggests that it is beneficial to have the two panels detuned in the sense that the radiating body fundamental natural frequency is significantly larger than that of the source body $(\beta \gg 1)$ in order to avoid a small denominator in the mean squared velocity expression. In fact, this is often the case in large passenger aircraft designs where the aircraft skin is made of aluminium alloy panels about $1-2 \mathrm{~mm}$ thick and the interior trim panels are made of polymer honeycomb sandwich of about $3-5 \mathrm{~mm}$ thickness that have a large stiffness to mass ratio. Referring again to Eq. (52), the $\alpha$ term in the numerator suggests that it is useful to decrease the equivalent stiffness of the air cavity between the two panels by for example increasing the distance between them. This is because $\alpha$ is the dimensionless measure of the stiffness of the coupling spring $k_{2}$. In conclusion, the simulations carried out with the broadband point force excitation on the reduced order model indicates some basic physics with regard to the stability and performance of the feedback loop. These on one hand suggest large effectiveness of the considered active control method for source panels stiffer than the radiating panels. On the other hand, in situations where the source panels are limp, it might be useful to consider the entirely passive control approach in particular if the two panels can be detuned in terms of their fundamental natural frequencies (avoiding $\beta \approx 1$ ).

\subsubsection{Turbulent boundary layer excitation}

In this section, the performance of the control system in reducing the TBL-induced sound transmission is studied by using the simplified, reduced order model. The modal expansion process of Section 2 is simplified by using only one fundamental mode per panel, $(1,1)$, and each cavity, $(0,0,0)$, in Eq. (10)-27).

The frequency- and space-averaged pressure PSD in the cavity $c_{2}$ and velocity PSD of the radiating panel can be obtained by numerically integrating Eq. 26) and Eq. (27), respectively, over frequencies up to the frequency of $1000 \mathrm{~Hz}$. The frequency- and space-averaged pressure and velocity PSD ( $I_{c_{2}, T B L}$ and $I_{p_{r}, T B L}$, respectively) are expressed as a function of the active and passive damping ratios. The passive and active damping ratios are calculated according to Eq. (34d) and Eq. 34e where the reference damping coefficient is that of the passive damper $c_{p}$. The results are organised and presented as follows: the frequency- and space-averaged velocity PSD of the radiating panel is shown in Fig. 77 a for $\beta>1$ and Fig. 77-b for $\beta<1$; the frequencyand space-averaged pressure PSD in the cavity $c_{2}$ is shown in Fig. 8 a for $\beta>1$ and Fig. 8-b for $\beta<1$ as a function of the passive and active damping ratios.

It is seen in the figures that again there is an optimal combination of the active and passive damping ratios, indicated with the white asterisk “*”, which minimises either the kinetic energy of the radiating panel, as shown by the white dash-dotted line in Fig. 7, or the frequency- and spaceaveraged pressure PSD in the cavity $c_{2}$, as shown in Fig. 8. Note that the optimal control parameters for the minimum sound pressure and the minimum kinetic energy are similar but not exactly the same. In fact, given the two mode approximation, where the acoustics of the back cavity $c_{2}$ is modelled using only the fundamental $(0,0,0)$ mode, minimisation of the space averaged pressure PSD in the back cavity would correspond to the minimisation of the space averaged displacement PSD of the radiating panel. As with the point force excitation, the radiating panel kinetic energy decreases monotonically with $\xi$ when $\beta<1$ Fig. 가(b) and Fig. 88-(b). Again, the active damping 
ratios $\xi$, corresponding to the optimal passive damping ratio $\eta_{o p t}$, is set to 10 and it is marked by the white asterisk in both figures. For $\beta>1$, the radiating panel vibrations increase as the stability limits, $\xi_{1}$ and $\xi_{2}$, are reached, Fig. 7 7. (a), leading to an increase of the interior sound pressure in the cavity $c_{2}$, Fig. 8. (a).

In addition, Fig. 9 shows the velocity PSD of the radiating panel as a function of frequency for cases $\beta>1$ and $\beta<1$. Finally, Fig. 10 shows the corresponding pressure PSD in the cavity $c_{2}$ for both cases $\beta>1$ and $\beta<1$.

As in the previous section, two approaches are considered in Fig. 9]10 (1) a fully passive approach (blue dotted line) achieved using an optimal tuned damping coefficient indicated by the

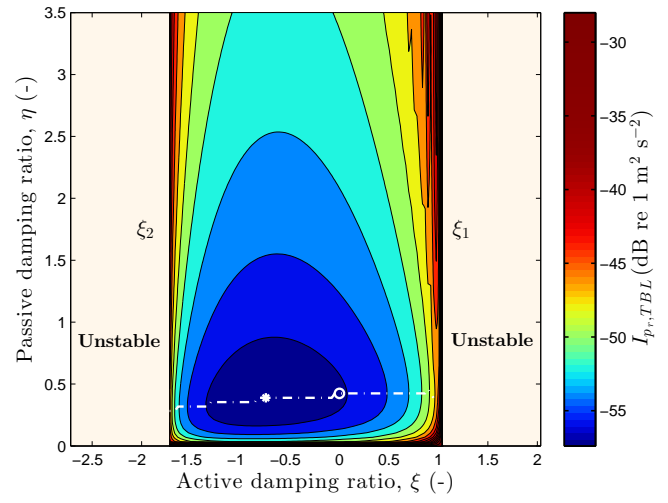

(a)

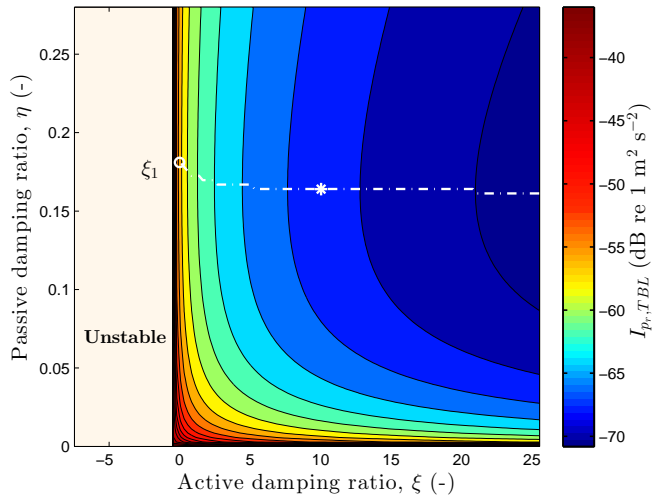

(b)

Figure 7: Frequency- and space-averaged velocity PSD of the radiating body plotted versus $\xi$ and $\eta$ with a TBL excitation, with $\eta_{\text {opt }}(\xi=0)$ (white $\left.\circ\right): \beta>1$ with $\left(\xi_{\text {opt }}, \eta_{\text {opt }}\right)$ (white $\left.*\right)(\mathrm{a}) ; \beta<1$ with $\left(\xi=10, \eta_{\text {opt }}(\xi=10)\right.$ ) (white $*$ ) (b).

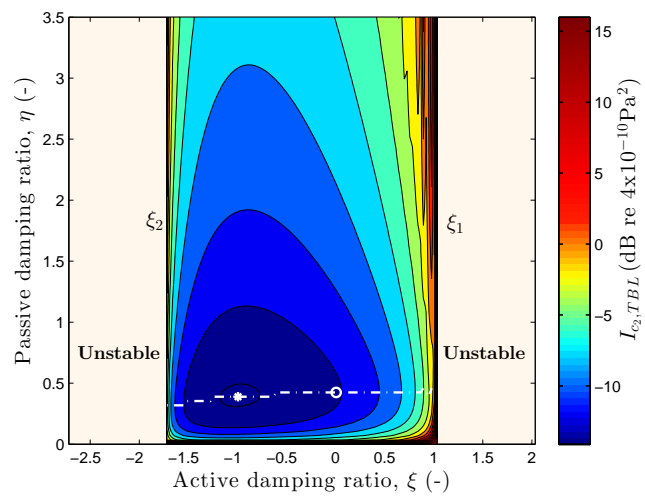

(a)

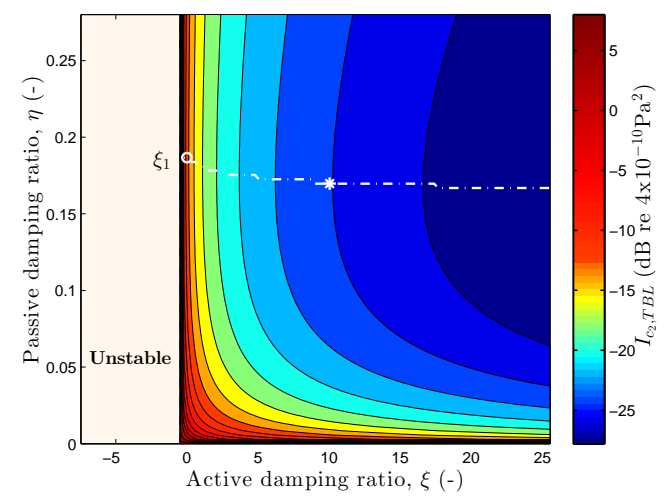

(b)

Figure 8: Frequency- and space-averaged pressure PSD in the cavity $c_{2}$ plotted versus $\xi$ and $\eta$ with a TBL excitation, with $\eta_{o p t}(\xi=0)$ (white $\left.\circ\right)$ : $\beta>1$ with $\left(\xi_{o p t}, \eta_{o p t}\right)($ white $*)(\mathrm{a}) ; \beta<1$ with $\left(\xi=10, \eta_{o p t}(\xi=10)\right)$ (white $\left.*\right)(\mathrm{b})$. 
circle in Fig. 7] 8, (2) a combination of active and passive damping (red solid line) achieved with the optimally tuned damping ratio $\left(\xi_{\text {opt }}, \eta_{\text {opt }}\right)$ indicated by the white asterisk in Fig. 7 - a and Fig. 85-a for $\beta>1$ and by choosing $\xi$ equal to 10 with $\eta$ calculated according to Eq. (48) in Fig. (7)-b and Fig. 8 -b for $\beta<1$. Also the case with the passive damping ratio of one tenth of the optimal is shown for comparison (red dash-dotted line in Fig. 96 b and Fig. 10-b) in the active case.

By comparing the situation with the TBL excitation to the scenario with the white noise force excitation discussed in the previous subsection, it can be stated that the stochastic TBL pressure distribution results in a steeper high frequency roll-off of the velocity PSD, shown in Fig. 9. An improvement due to the optimal active control, in comparison with the passive one, is still found;

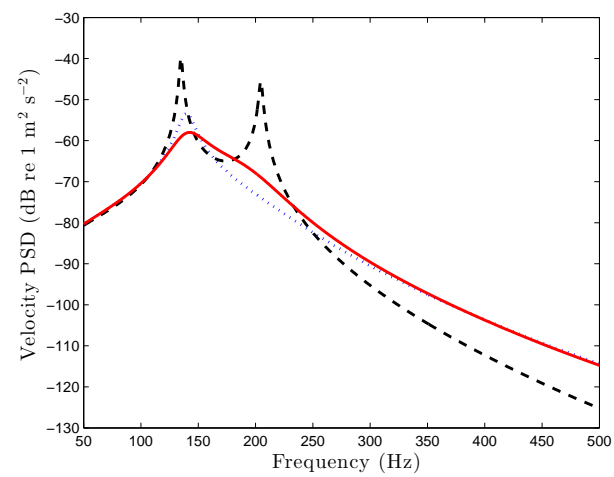

(a)

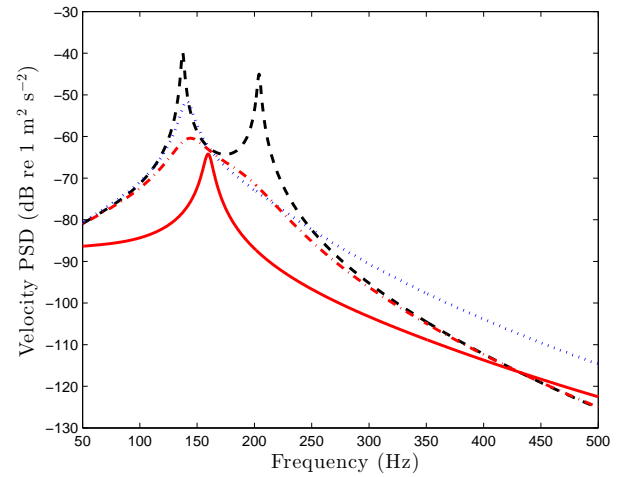

(b)

Figure 9: Velocity PSD of the radiating body for a TBL excitation: $\beta>1$ (a); $\beta<1$ (b). No control (--), passive control with $\eta_{\text {opt }}(\xi=0)(\cdots)$, optimal tuned active control with $\left(\xi_{o p t}, \eta_{o p t}\right)$ for $\beta>1$ and with $\left(\xi=10, \eta_{o p t}(\xi=10)\right)(-)$ and $\left(\xi=10, \eta=\eta_{\text {opt }} / 10\right)(-\cdot-)$ for $\beta<1$.

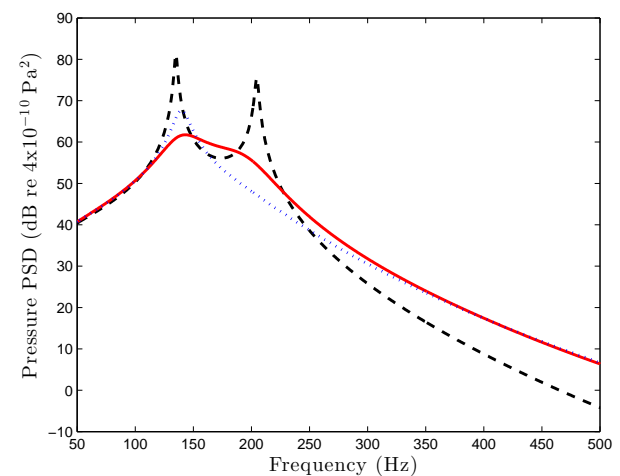

(a)

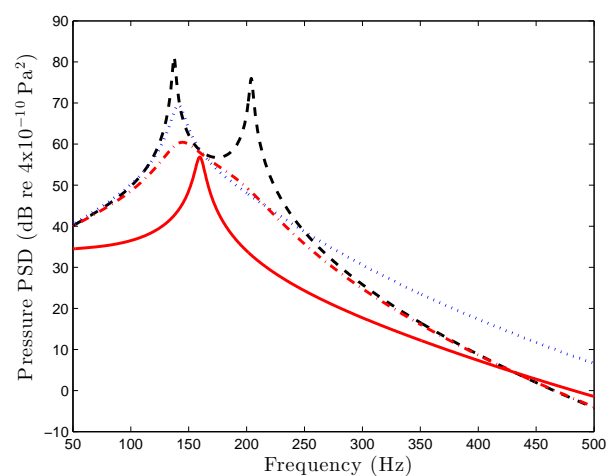

(b)

Figure 10: Pressure PSD in the cavity $c_{2}$ for a TBL excitation: $\beta>1$ (a); $\beta<1$ (b). No control (--), passive control with $\eta_{\text {opt }}(\xi=0)(\cdots)$, optimal tuned active control with $\left(\xi_{\text {opt }}, \eta_{\text {opt }}\right)$ for $\beta>1$ and with $\left(\xi=10, \eta_{\text {opt }}(\xi=10)\right)(-)$ and $\left(\xi=10, \eta=\eta_{\text {opt }} / 10\right)(-\cdot-)$ for $\beta<1$. 
even when the passive damping ratio is one tenth of the optimal one. Indeed, minimizing the kinetic energy (or velocity PSD) of the radiating panel corresponds to the situation shown by the red solid lines in Fig. 9.b and Fig. 10-b. This requires careful tuning of the passive and active damping ratios to the optimum combination. In our case, the relatively large optimal passive damping ratio results in an "overshot" at frequencies above approximately $430 \mathrm{~Hz}$ when compared to the situation without any control. Hence, the relevance of the example with the passive damping ratio of the $1 / 10$ of the optimal one is to show that the "overshot" is due to a relatively large passive damping ratio, and, that it can be moved to a larger frequency by decreasing the passive damping ratio (i.e. the dash-dotted red line does not go over the black dashed line up to at least $500 \mathrm{~Hz}$ ). Therefore, if necessary, the overshot effect can be minimized by using a passive damping ratio smaller than the optimal one. This would be, however, on the expense of the low frequency control of the vibration transmission.

To conclude, also with a TBL excitation, the proposed control approach (either active or passive) gives the expected, if not better, results.

\section{Results with the full order model}

In this section, results of simulations using a total number of $\mathrm{M}_{p_{s}}=24$ and $\mathrm{M}_{p_{r}}=20$ panel modes, and, $\mathrm{N}_{c_{1}}=24$ and $\mathrm{N}_{c_{2}}=48$ acoustic modes are presented. The stability and performance of the active control system are again discussed in terms of the mean squared vibration velocity of the radiating panel and the mean squared acoustic pressure in the cavity $c_{2}$. The number of modes used enables accurate calculation of the mean squared pressure and velocity up to $1000 \mathrm{~Hz}$. Two load conditions are analysed: 1) white noise point force excitation; and 2) TBL excitation. The large number of modes precludes the use of the Routh-Hurwitz criterion to assess the stability of the active system such that the Nyquist criterion is used instead.

\subsection{Stability}

The sensor-actuator OL - FRF can be used to analyse the stability of the closed loop control system by using the Nyquist criterion. A total number of $\mathrm{M}_{p}=60$ panel modes, and, $\mathrm{N}_{c_{1}}=$ 162 and $\mathrm{N}_{c_{2}}=297$ acoustic modes are considered for a frequency range up to $5 \mathrm{kHz}$. The sensor-actuator OL - FRF for the control system at hand is the frequency response function, $y_{s, a}$, between the error velocity measured by the sensor located on the radiating panel, $p_{r}$, which is due to the actuator force, $f_{c}$, when the primary excitation is switched off. In Fig. 11]- 12, the corresponding Bode and Nyquist plots are shown. Fig. 11] is for the case with $\beta>1$ and Fig. 12 is for the case $\beta<1$.

For $\beta>1$, it is seen in the Nyquist plot that the locus of the OL - FRF crosses the negative real axis. Bode plots show that the crossover occurs near the first natural frequency of the coupled system at $135 \mathrm{~Hz}$. In fact, the amplitude of the sensor-actuator OL - FRF has a low-frequency antiresonance followed by two consecutive resonances. Also the phase undergoes first a lead and then two consecutive lags. This lead-lag pattern is an indication that the system characterized by $\beta>1$ is conditionally stable and the maximum feedback gain is limited. The amplitude of the sensor-actuator OL - FRF at the crossover frequency is about $0.09 \mathrm{~m} \mathrm{~s}^{-1} \mathrm{~N}^{-1}$ so the maximum gain is about $11 \mathrm{~N} \mathrm{~s} \mathrm{~m}^{-1}$ above which the Nyquist point $(-1+0 \mathrm{i})$ would be encircled.

On the contrary, for $\beta<1$, the Nyquist plot is located entirely in the two positive real quadrants of the real-imaginary plane up to at least $5 \mathrm{kHz}$. The phase plot in the Bode diagram indicates that the phase of the sensor-actuator OL - FRF is bound between \pm 90 degrees. Between any 

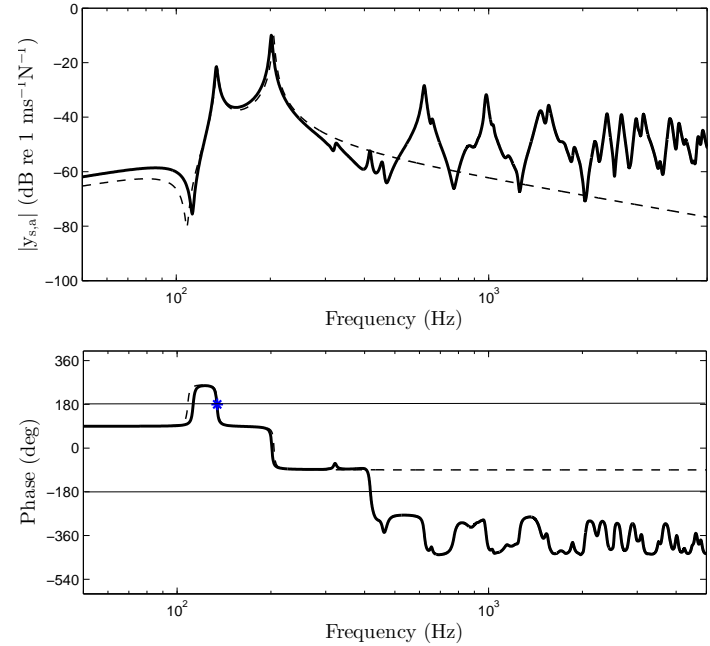

(a)

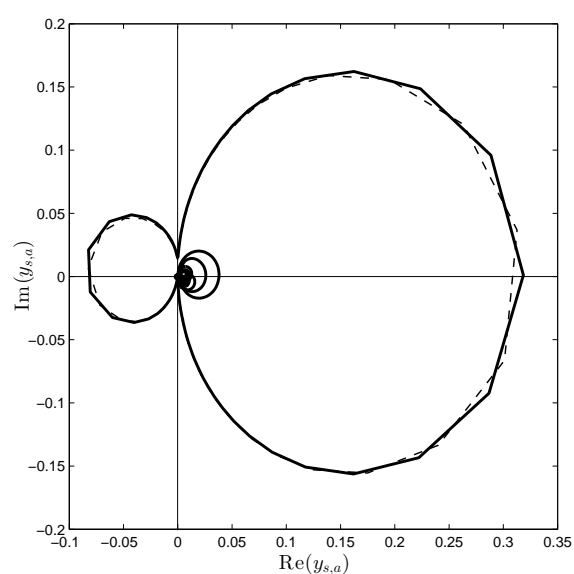

(b)

Figure 11: Case for $\beta>1$. Bode plots (a); Nyquist Plot of the open loop sensor-actuator FRF (b).
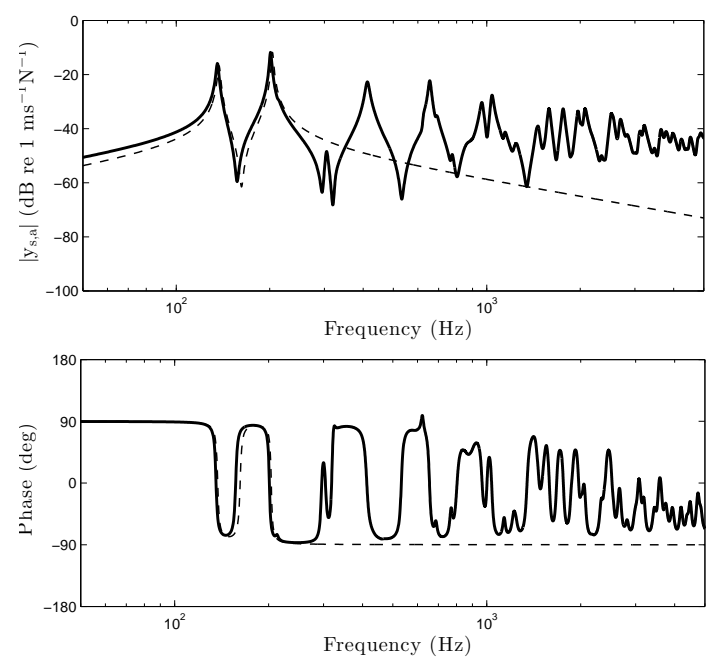

(a)

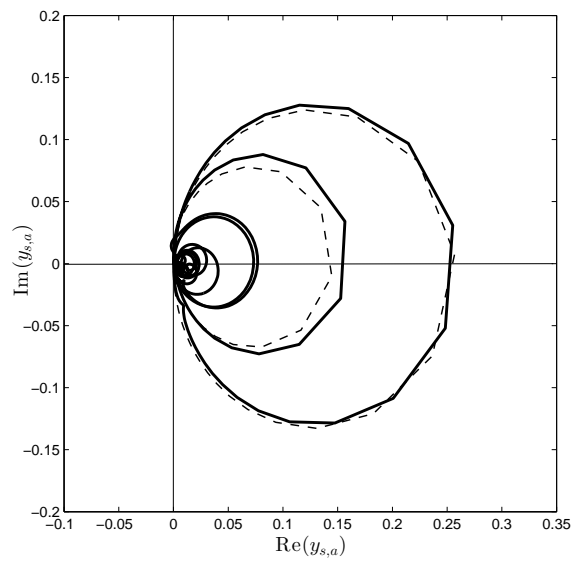

(b)

Figure 12: Case for $\beta<1$. Bode plots (a); Nyquist Plot of the open loop sensor-actuator FRF (b).

two resonances in the amplitude Bode plot an antiresonance can be found. Thus the system with $\beta<1$ is unconditionally stable under the assumption of ideal sensor-actuator frequency response. Further limitations to the maximum feedback gain can be expected in practice, due to the real dynamics of the sensor and the actuator and/or the non-perfect physical matching of the sensoractuator locations. Nevertheless, in case of $\beta<1$, the OL - FRF has desirable properties which indicate that good stability margins can also be expected in practice. 
Comparing now the present full order model stability analysis to the reduced order model one, a very similar behaviour is observed. The sensor-actuator OL - FRF obtained using the reduced two mode model is shown in Fig.11-12 using dashed lines which very well coincide with the full order model loci, amplitude, and phase at low frequencies near the crossover frequency. It thus appears that despite the fact that many high-order flexible modes contribute to the sensor-actuator OL - FRF, the fundamental behaviour is well captured by the reduced 2 mode model and the analytical stability limits calculated in Section 3.2 can be expected to hold in more realistic situations with many modes governing the vibroacoustic response of the system.

Regarding the high-frequency behaviour, it is still interesting that the phase of the OL - FRF is bound between \pm 90 degrees, given the fact that the sensor and the actuator are not dual and collocated. In order to explain this, it should be noted that the $y_{s, a}$ can be expressed as a sum of a collocated FRF between the component of the control force acting on the radiating panel and the sensor (driving point), and a non-collocated FRF between the component of the control force reacting against the source panel (transfer), Fig. 1] The non-collocated FRF, being a transfer mobility, rolls-off with frequency with $-20 \mathrm{~dB}$ per decade, whereas the collocated FRF does not roll-off (tends to a pure real number as the frequency tents to infinity). This is shown in Fig. 13. Therefore the relative importance of the non-collocated FRF decreases as the frequency increases.

\subsection{Performance}

In the following subsection, the performance of the control system assuming a full order model is analysed for both load conditions: point force and TBL. The frequency-averaged vibration velocity of the radiating panel and the sound pressure in the acoustic enclosure $c_{2}$ are used as the metrics for the quality of the sound transmission control. The performance of the control system is again discussed as a function of $\xi$ and $\eta$, Eq. 34e.

\subsubsection{Point force excitation}

In the case of a point force excitation on the radiating panel, the mean squared velocity at the centre of the radiating panel and the mean squared acoustic pressure near the corner of

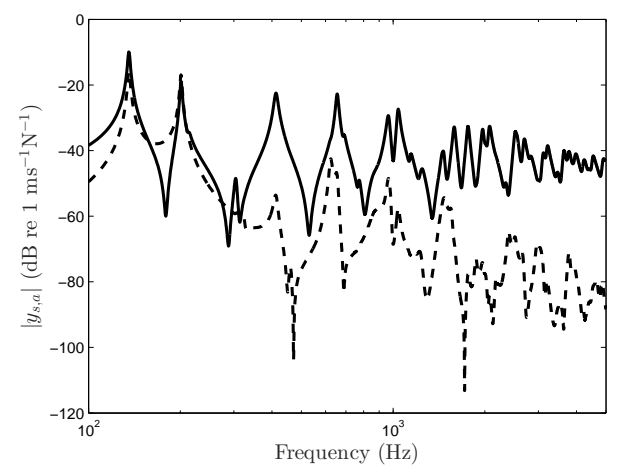

(a)

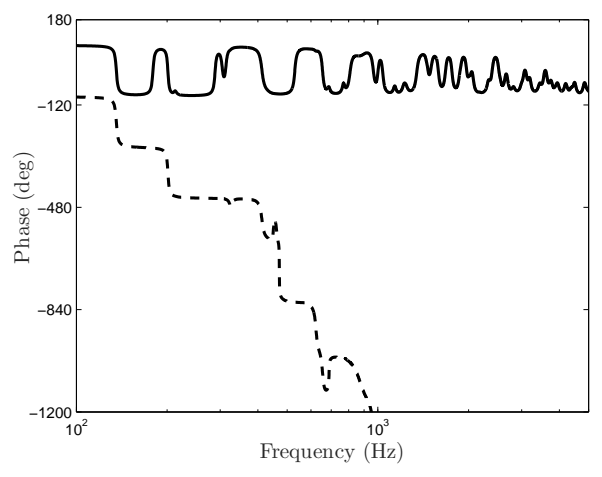

(b)

Figure 13: Case for $\beta<1$. Amplitude (a) and phase (b) of the collocated (solid line) and non-collocated (dashed line) transfer mobility. 
the cavity $c_{2}$ are calculated using the procedure described in Section 2.2 The location of the pressure monitoring point is $\left(x_{p}, y_{p}, z_{p}\right)=\left(L_{c_{x}} / 3, L_{c_{y}} / 3,-2 L_{c_{2}} / 3\right)$. And, the location of the point force exciting the source panel is $\left(x_{f}, y_{f}\right)=(a / 3, b / 3)$. The squared magnitude of either frequency response function is integrated numerically over the frequency range $0-1000 \mathrm{~Hz}$. The corresponding mean squared velocity and pressure are shown in Fig. 14 - a and Fig. 15-a, respectively, for the case $\beta<1$ only. The amplitude of the velocity at the centre of the radiating panel per unit excitation force on the source panel is shown in Fig. 14 - b, and the amplitude of the sound pressure per unit excitation force at the pressure monitoring point in the cavity $c_{2}$ is shown in Fig. 15-b. Again, the results with the active approach are contrasted to the results using a fully passive approach.

It can be seen that the vibrations of the radiating panel decrease with an increase in the active damping ratio. A similar behaviour is observed for the sound pressure at the corner of the cavity $c_{2}$. Large values of $\xi$ can yield noteworthy sound transmission control effects. As a result, significant improvements over the passive control due to the active control can be seen in the amplitude plots of either the radiating panel velocity or the sound pressure in the back cavity.

\subsubsection{Turbulent boundary layer excitation}

For the full order model assuming the TBL excitation on the source panel, the performance of the control system is analysed in terms of: a) the velocity PSD at the centre of the radiating panel, $p_{r}$, and $\mathrm{b}$ ) the pressure PSD near the corner of the cavity $c_{2}$, see Fig. 11. (The location of the sound pressure monitoring point is the same as in the previous subsection.) The corresponding results are shown in Fig. 16-17]for the case $\beta<1$ and in Fig. 18-19]for the case $\beta>1$.

Again, some frequency-averaged control performance metrics are used. To this end, the pressure PSD, Eq. 26, near the corner of the cavity $c_{2}$ and the velocity PSD, Eq. 27] at the centre of the radiating panel are integrated numerically over the frequency range $0-1000 \mathrm{~Hz}$. It can be seen in Fig. 16-a and 18- a that the broadband noise reduction performance is qualitatively similar to

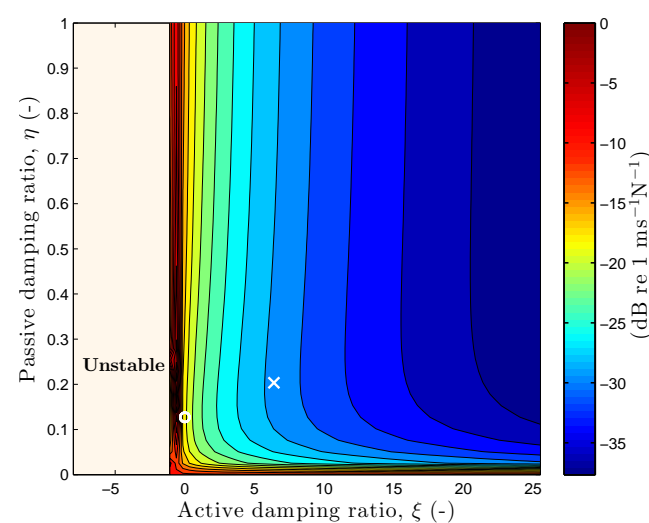

(a)

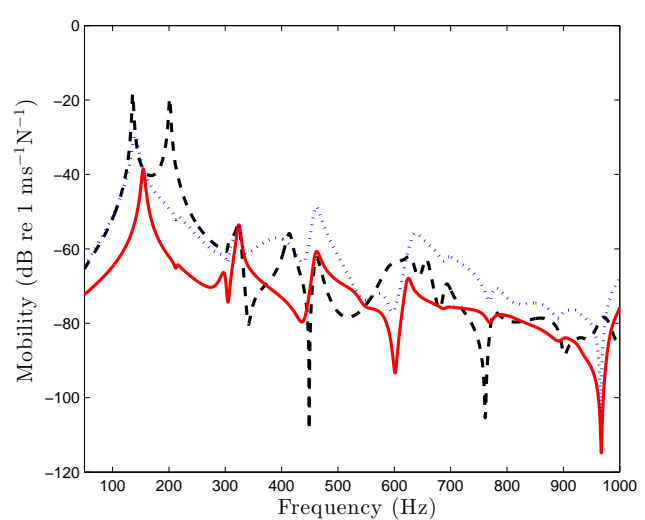

(b)

Figure 14: Mean squared velocity of the radiating panel plotted versus $\xi$ and $\eta$ in case of a point force excitation for $\beta<1$, with $\eta_{\text {opt }}(\xi=0)$ (white $\circ$ ) and $\left(\xi=6.3, \eta_{\text {opt }}(\xi=6.3)\right.$ ) (white $\times$ ) (a). Amplitude of the velocity at the centre of $p_{r}$ : no control $(--)$; passive control with $\eta_{o p t}(\xi=0)(\cdots)$, optimal tuned active control with $\left(\xi=6.3, \eta_{o p t}(\xi=6.3)\right)(-)$ for $\beta<1$ (b). 


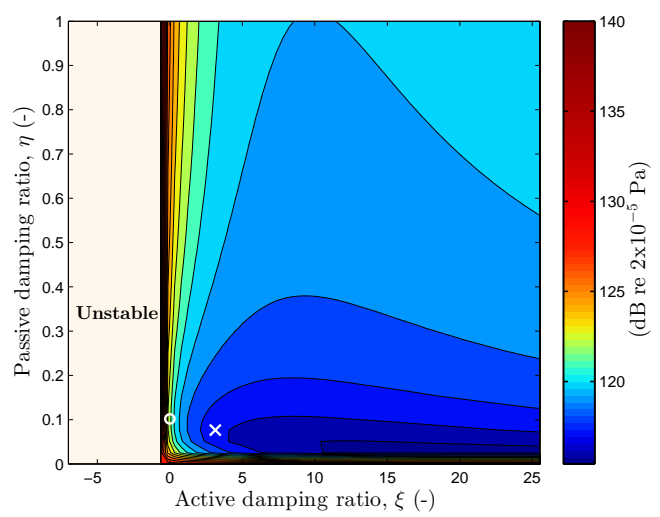

(a)

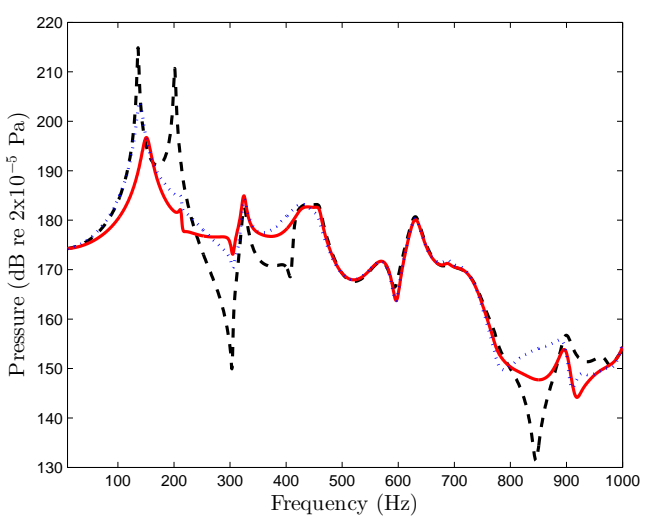

(b)

Figure 15: Mean squared pressure in the cavity $c_{2}$ plotted versus $\xi$ and $\eta$ in case of a point force excitation for $\beta<1$, with $\eta_{\text {opt }}(\xi=0)$ (white $\circ$ ) and $\left(\xi=3.13, \eta_{\text {opt }}(\xi=3.13)\right.$ ) (white $\left.\times\right)$ (a). Sound pressure in the cavity $c_{2}$ : no control (--); passive control with $\eta_{o p t}(\xi=0)(\cdots)$; optimal tuned active control with $\left(\xi=3.13, \eta_{\text {opt }}(\xi=3.13)\right)(-)$ for $\beta<1$ (b) at $\left(x_{p}, y_{p}, z_{p}\right)=\left(L_{c_{x}} / 3, L_{c_{y}} / 3,-2 L_{c_{2 z}} / 3\right)$.

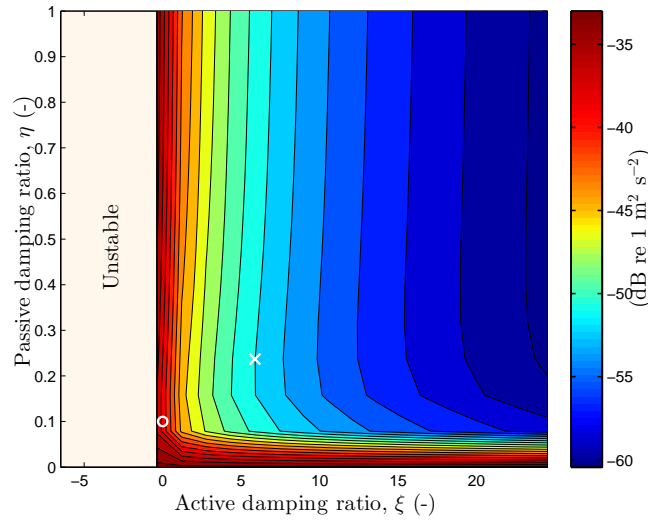

(a)

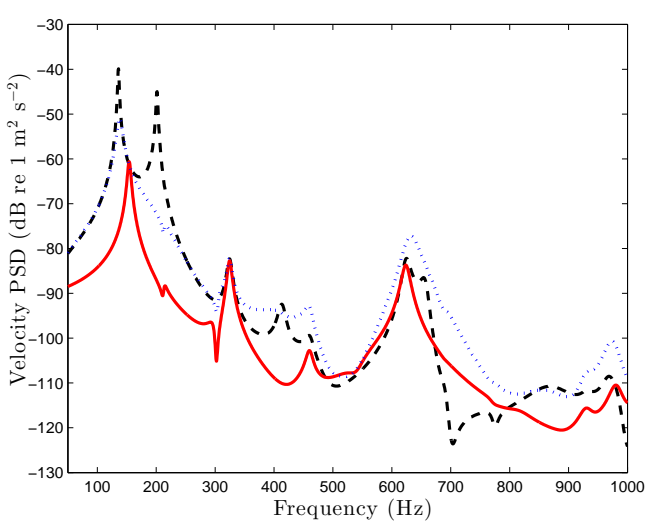

(b)

Figure 16: Frequency-averaged velocity PSD at the centre of $p_{r}$, for a TBL excitation, plotted versus $\xi$ and $\eta$ for $\beta<1$, with $\eta_{o p t}(\xi=0)$ (white $\circ$ ) and $\left(\xi=6.3, \eta_{o p t}(\xi=6.3)\right.$ ) (white $\left.\times\right)$ (a). Velocity PSD at the centre of $p_{r}$, for $\beta<1$ : no control (--); passive control with $\eta_{\text {opt }}(\xi=0)(\cdots)$; optimal tuned active control with $\left(\xi=6.3, \eta_{o p t}(\xi=6.3)\right)(-)(\mathrm{b})$.

that observed with the reduced order model (Fig. 7). In Fig. 16-a and Fig. 17.a, for $\beta<1$, it can be seen that the frequency averaged velocity and pressure PSDs, respectively, decrease with increasing the gain $g$ (or $\xi$ ). On the other side, if $\beta>1$, the frequency averaged pressure increases as $\xi$ moves closer to the stability limits as shown in Fig. 18-a and Fig. 19. a.

Note that for the full order model the stability limits for $\xi$ are changing with the passive damping ratio as it is shown in the plots of Fig. 18. a and Fig. 19. a. This behaviour is also shown in Fig. 
20. where the stability limits of the control system are plotted for different structural damping ratios of the panels. In Fig. 20 it is shown that when the structural damping ratio of the panels is reduced the stability limits tend to $\xi_{1}$ and $\xi_{2}$ found for the reduced order model, see Section 3.2 The pressure PSD near the corner of $c_{2}$ is plotted versus frequency in Fig. 17. b with $\beta<1$ and Fig. 19.b with $\beta>1$ for three cases: without control, with passive control using $c_{p}$ only, and finally with active control using both $c_{p}$ and $g$. Similar plots are shown for the velocity PSD at

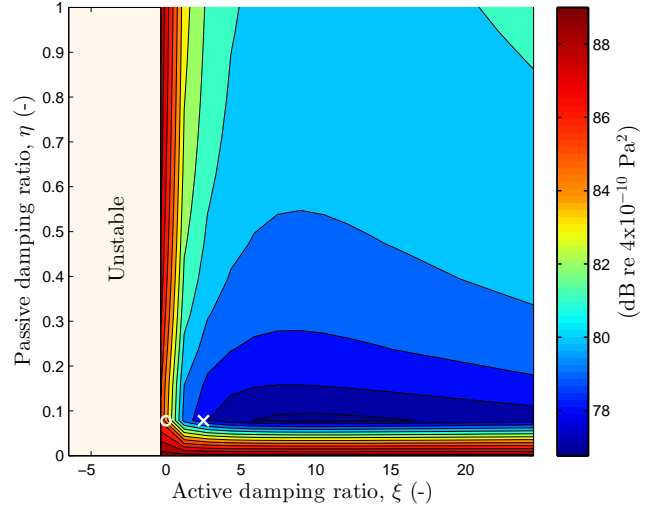

(a)

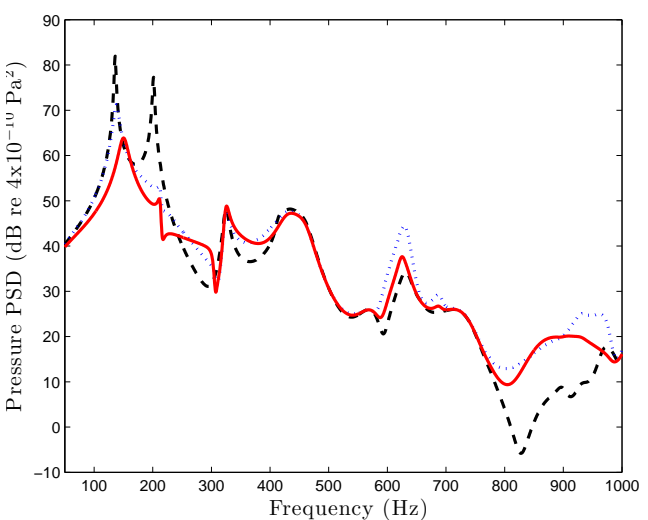

(b)

Figure 17: Frequency-averaged pressure PSD in $c_{2}$, for a TBL excitation, plotted versus $\xi$ and $\eta$, for $\beta<1$, with $\eta_{o p t}(\xi=0)$ (white $\circ$ ) and $\left(\xi=3.13, \eta_{o p t}(\xi=3.13)\right.$ ) (white $\left.\times\right)$ (a). Pressure PSD in $c_{2}$ for $\beta<1$ : no control (--); passive control with $\eta_{o p t}(\xi=0)(\cdots)$; optimal tuned active control with $\left(\xi=3.13, \eta_{o p t}(\xi=3.13)\right)(-)$ (b) at $\left(x_{p}, y_{p}, z_{p}\right)=$ $\left(L_{c_{x}} / 3, L_{c_{y}} / 3,-2 L_{c_{2 z}} / 3\right)$.

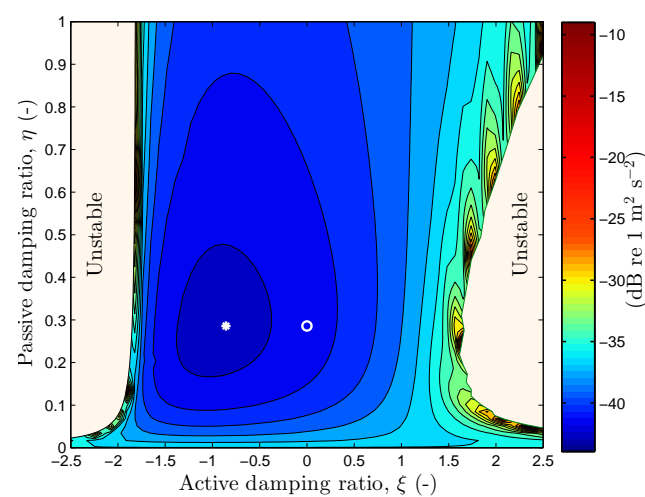

(a)

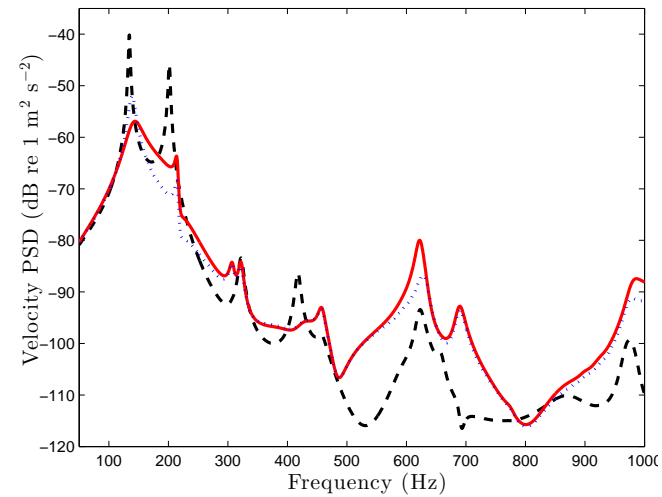

(b)

Figure 18: Frequency-averaged velocity PSD at the centre of $p_{r}$, for a TBL excitation, plotted versus $\xi$ and $\eta$ for $\beta>1$, with $\eta_{o p t}(\xi=0)$ (white $\circ$ ) and $\left(\xi_{o p t}, \eta_{o p t}\right)$ (white $\left.*\right)$ (a). Velocity PSD at the centre of $p_{r}$, for $\beta>1$ : no control (--); passive control with $\eta_{\text {opt }}(\xi=0)(\cdots)$; optimal tuned active control with $\left(\xi_{\text {opt }}, \eta_{\text {opt }}\right)(-)(\mathrm{b})$. 


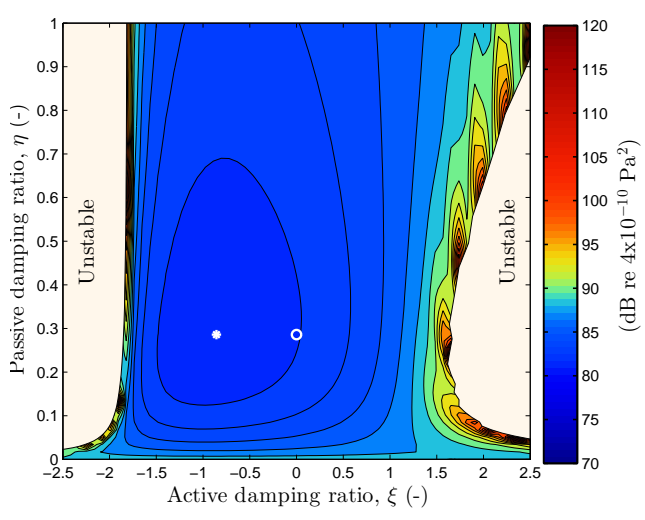

(a)

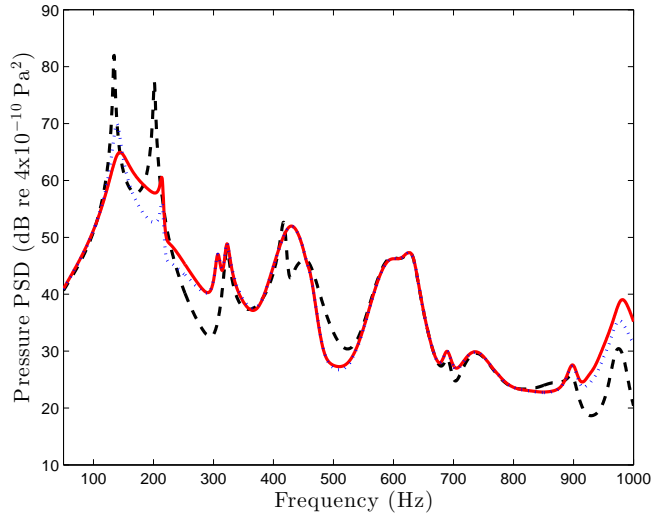

(b)

Figure 19: Frequency-averaged pressure PSD in $c_{2}$, for a TBL excitation, plotted versus $\xi$ and $\eta$, for $\beta>1$, with $\eta_{o p t}(\xi=0)$ (white $\circ$ ) and $\left(\xi_{\text {opt }}, \eta_{o p t}\right)$ (white $\left.*\right)(\mathrm{a})$. Pressure PSD for $\beta>1$ : no control (--); passive control with $\eta_{o p t}(\xi=0)(\cdots)$; optimal tuned active control with $\left(\xi_{o p t}, \eta_{o p t}\right)(-)(\mathrm{b})$ at $\left(x_{p}, y_{p}, z_{p}\right)=\left(L_{c_{x}} / 3, L_{c_{y}} / 3,-2 L_{c_{2 z}} / 3\right)$.

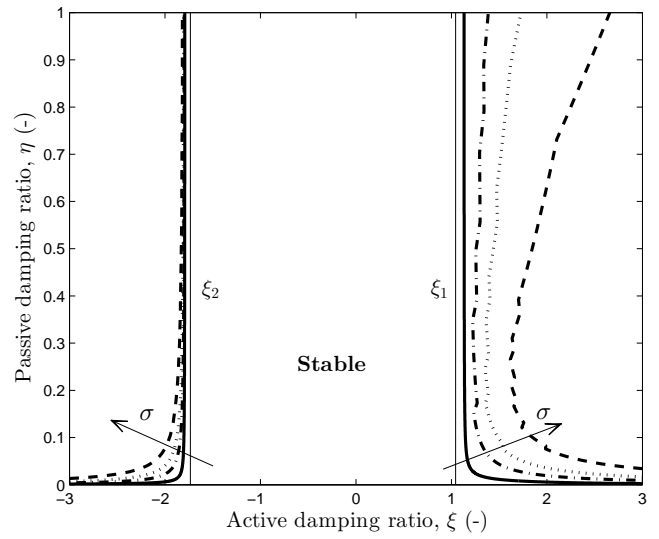

Figure 20: Stability limit for $\beta>1$ and for different structural damping ratio: $\sigma=0.01(--), \sigma=0.005(\cdots), \sigma=0.0025$ $(-\cdot-)$ and $\sigma=0.0005(-)$.

the centre of the radiating panel in Fig. 16-b with $\beta<1$ and Fig. 18.b with $\beta>1$. For the passive control, the optimally tuned damping coefficients given by the hollow circles in Fig. 16, 19. a are used. For the active control case with $\beta<1$ (red curves in Fig. 16, 17.b), the combination of active and passive damping ratios used is indicated by the white $\times$ in Fig. 16 17. a. For the active control case with $\beta>1$ (red curves in Fig. 18 19.b), the optimal combination of active and passive damping ratios used, $\left(\xi_{\text {opt }}, \eta_{\text {opt }}\right)$, is given by the minimum frequency-averaged velocity PSD at the centre of the radiating panel and the minimum frequency-averaged pressure PSD in the back cavity $c_{2}$, and, it is designated by the white $*$ in Fig. 18 19 . a.

Once again, the case $\beta<1$ results in better sound transmission reduction effects with the active 
control approach. At particular higher frequencies there can be a slight increase when the control is set on indicating a control spillover, as shown in Fig. 16]19-b. This increase is particularly marked in the case $\beta>1$.

Nevertheless, large reductions of the interior sound levels can be expected at the lowest resonance frequencies. By comparing the right-hand side plot of Fig. [16 to the right hand side plot of Fig. 177, it can be concluded that large contributions to the interior sound pressure are due to the two lowest double panel modes. The relative contribution of the two modes to the total audible interior sound may however be less significant if the human perception of sound is taken into account (for example A-weighting).

Note that, for the full order model, the pressure distribution in the two cavities is not uniform. Instead, the acoustic field in the cavities is influenced by the motion of the panels. In Appendix D additional mode shapes obtained using the full order model are shown and discussed. The two fundamental modes contribute most to both the vibration of the radiating panel and the sound pressure inside the cavity $c_{2}$ as seen in Fig. 14, 15] as well as in Fig. 16, 17] In fact, the second fundamental mode corresponds to the so-called mass-air-mass resonance frequency [11], above which the spectra of either radiating panel velocity or the sound pressure roll-off steeply with frequency. This is why controlling vibrations of the radiating panel due to the first two fundamental modes gives significant reductions of the sound pressure in the cavity $c_{2}$.

Further work will address the inclusion of the sensor-actuator dynamics and experimental validation of the active control of TBL-induced noise transmission through the double leaf partition.

\section{Conclusions}

In this paper, the active control of TBL noise transmission through a cavity-backed double panel is investigated. Stability and performance of the velocity feedback active control system are carried out for a reduced order model and an increased order model. The theoretical analysis indicates that the first fundamental mode and the mass-air-mass mode of the coupled system are the strongest radiators of sound into the back cavity. Additionally, the two modes mark the dynamics of the coupled system in such a way that it is possible to derive the stability limits of the active control system using only these two modes within the reduced order model. Closed form expressions for these stability limits are given in terms of the minimal/maximal active damping ratio. Similar limits are found for the increased order model, which only slightly changes with the passive damping ratio. Behaviour of the control system strongly depends on the ratio for the fundamental resonance frequencies of the two panels. The fundamental resonance frequency of the source panel is larger than the fundamental resonance frequency of the radiating panel, which results in unconditionally stable control systems such that very large feedback gains can be used. Consequently, the simplified model considers the cross coupling effects for a stationary TBL excitation, which is useful to evaluate the performance of feedback control systems when the active reductions of the low frequency TBL noise transmission through multiple aircraft panels are needed. Also, large sound pressure reductions in a broad frequency band in the acoustic back cavity can be expected. In case when the fundamental natural frequency of the source panel is lower than that of the radiating panel, the feedback loop is conditionally stable so that the maximum feedback gain is limited. Then the performance of the active control is comparable to that of a passive, optimally tuned viscous damper with its terminals mounted onto the source and radiating panels. 


\section{Acknowledgements}

The research work of A. Caiazzo has been funded by the European Commission within the ITN Marie Curie Action project BATWOMAN under the 7th Framework Programme (EC grant agreement no. 605867). This research was partially supported by Flanders Make, the strategic research centre for the manufacturing industry. This project has received funding from the European Union's Horizon 2020 research and innovation programme under the Marie SklodowskaCurie grant agreement no. 657539. The Research Fund KU Leuven is also gratefully acknowledged for its support.

\section{Appendix A. Analytical expressions for the matrices M, D and K (15)}

The expressions for the mass, damping and stiffness matrices of the coupled system analysed in Section 2.2 are summarized as follows,

$$
\begin{gathered}
\mathbf{M}_{i i}=\operatorname{diag}[\rho t]_{i}, \quad \mathbf{M}_{c c}=\operatorname{diag}\left[\frac{1}{c_{0}^{2}}\right]_{c}, \\
\mathbf{D}_{c c}=\operatorname{diag}\left[2 \frac{1}{c_{0}^{2}} \omega_{n} \sigma_{a c}\right]_{c}, \\
\mathbf{K}_{i i}=\operatorname{diag}\left[\omega_{m}^{2} \rho t\right]_{i}, \quad \mathbf{K}_{c c}=\operatorname{diag}\left[\frac{\omega_{n}^{2}}{c_{0}^{2}}\right]_{c},
\end{gathered}
$$

in which $\omega_{n}$ is the natural frequency of the acoustic $c$-enclosure. In Eq. A.1] and A.3), $\mathbf{M}_{i i}$ and $\mathbf{K}_{i i}$ are the mass and stiffness matrices of the $i$-panel, with $i=p_{s}, p_{r}$ for the source and radiating panel, respectively. And $\mathbf{M}_{c c}, \mathbf{D}_{c c}$ and $\mathbf{K}_{c c}$ are the mass, damping and stiffness matrices for the $c$-enclosure, with $c=c_{1}, c_{2}$ for the cavity of the double panel system and the back cavity, respectively, as shown in Fig. 1. The matrices $\mathbf{M}_{c i}$ and $\mathbf{K}_{i c}$ in Eq. (15) represent the coupling between the structural vibration and the enclosure acoustic pressure, and they are given by

$$
\begin{aligned}
& \mathbf{M}_{c_{1} p_{s}}=\rho_{0}\left[\frac{(-1)^{n_{z}} A_{n_{z}}}{\sqrt{L_{c_{1_{z}}}}} \int_{0}^{a} \int_{0}^{b} \psi_{p_{s_{m_{x} m_{y}}}}(x, y) \gamma_{c_{1_{n_{x} n_{y}}}}(x, y) \mathrm{dx} \mathrm{dy}\right], \\
& \mathbf{M}_{c_{1} p_{r}}=\rho_{0}\left[-\frac{A_{n_{z}}}{\sqrt{L_{c_{1_{z}}}}} \int_{0}^{a} \int_{0}^{b} \psi_{p_{r_{m_{x}} m_{y}}}(x, y) \gamma_{c_{n_{n_{x} n_{y}}}}(x, y) \mathrm{dxdy}\right] \text {, } \\
& \mathbf{M}_{c_{2} p_{r}}=\rho_{0}\left[\frac{A_{n_{z}}}{\sqrt{L_{c_{2}}}} \int_{0}^{a} \int_{0}^{b} \psi_{p_{r_{m_{x} m_{y}}}}(x, y) \gamma_{c_{2_{n_{x} n_{y}}}}(x, y) \mathrm{dxdy}\right] \text {, } \\
& \mathbf{K}_{p_{s} c_{1}}=\left[-\frac{(-1)^{n_{z}} A_{n_{z}}}{\sqrt{L_{c_{z}}}} \int_{0}^{a} \int_{0}^{b} \psi_{p_{s_{m_{x} m_{y}}}}(x, y) \gamma_{c_{n_{n_{x}} n_{y}}}(x, y) \mathrm{dxdy}\right], \\
& \mathbf{K}_{p_{r} c_{1}}=\left[\frac{A_{n_{z}}}{\sqrt{L_{c_{1_{z}}}}} \int_{0}^{a} \int_{0}^{b} \psi_{p_{r_{m_{x} m_{y}}}}(x, y) \gamma_{c_{1_{n_{x} n_{y}}}}(x, y) \mathrm{dxdy}\right] \text {, } \\
& \mathbf{K}_{p_{r} c_{2}}=\left[-\frac{A_{n_{z}}}{\sqrt{L_{c_{2 z}}}} \int_{0}^{a} \int_{0}^{b} \psi_{p_{r_{m_{x}} m_{y}}}(x, y) \gamma_{c_{2_{n_{x} n_{y}}}}(x, y) \mathrm{dxdy}\right] \text {, }
\end{aligned}
$$


with $\psi_{i_{m_{x} m_{y}}}(x, y)$ defined in Eq. (12) and

$$
\gamma_{c_{n_{x} n_{y}}}(x, y)=\frac{A_{n_{x}} A_{n_{y}}}{\sqrt{L_{c_{x}} L_{c_{y}}}} \cos \left(n_{x} \pi x / L_{c_{x}}\right) \cos \left(n_{y} \pi y / L_{c_{y}}\right) .
$$

Therefore, the analytical expressions for Eq. A.4 - A.5 are found by solving the integrals over the plate area, as shown in [36]. Note that, $\mathbf{M}_{c i} \in \mathbf{R}^{\mathbf{N}_{c} \times \mathbf{M}_{i}}, \mathbf{K}_{i c} \in \mathbf{R}^{\mathrm{M}_{i} \times \mathbf{N}_{c}}$, and $\mathbf{M}_{c c}, \mathbf{K}_{c c}, \mathbf{D}_{c c} \in$ $\mathbf{R}^{\mathbf{N}_{c} \times \mathbf{N}_{c}}$, and $\mathbf{M}_{i i}, \mathbf{K}_{i i} \in \mathbf{R}^{\mathbf{M}_{i} \times \mathbf{M}_{i}}$. Therefore, $\mathbf{M}, \mathbf{K}$ and $\mathbf{D} \in \mathbf{R}^{\left(\mathrm{M}_{p_{s}}+\mathrm{M}_{p_{r}}+\mathbf{N}_{c_{1}}+\mathrm{N}_{c_{2}}\right) \times\left(\mathrm{M}_{p_{s}}+\mathbf{M}_{p_{r}}+\mathbf{N}_{c_{1}}+\mathbf{N}_{c_{2}}\right)}$.

\section{Appendix B. Derivation of the equivalent coefficients $m_{1}, m_{2}, k_{1}, k_{2}$ and $k_{3}$}

The equivalent coefficients are obtained by imposing the equivalence between the components of the frequency response function matrix for the model shown in Section 2.2 when excited by a point force and the one of a known two degree of freedom lumped parameter mechanical system [24]. They equivalent system coefficients are:

$$
\begin{gathered}
m_{1}=\frac{t_{p s} \rho a b}{4 C_{2}}, \quad m_{2}=\frac{t_{p r} \rho a b}{4 C_{3}}, \\
k_{1}=\frac{D_{1} \pi^{4}\left(a^{2}+b^{2}\right)^{2}}{4 b^{3} a^{3} C_{2}}, \\
k_{2}=\frac{C_{4}-D_{1} D_{2} L_{c_{2}} \pi^{8}\left(a^{2}+b^{2}\right)^{2} C_{3}}{4 L_{c_{1}} \pi^{4} a^{3} b^{3}\left(D_{1}+D_{2}\right) C_{3} C_{2}}, \\
k_{3}=\frac{D_{2} \pi^{4}\left(a^{2}+b^{2}\right)^{2}}{4 b^{3} a^{3} C_{2}},
\end{gathered}
$$

where

$$
\begin{gathered}
D_{1}=\frac{E t_{p s}^{3}}{12\left(1-v^{2}\right)}, \quad D_{2}=\frac{E t_{p r}^{3}}{12\left(1-v^{2}\right)}, \\
C_{2}=\sin \left(\frac{\pi y_{f}}{b}\right) \sin \left(\frac{\pi x_{f}}{a}\right) \sin \left(\frac{\pi y_{s}}{b}\right) \sin \left(\frac{\pi x_{s}}{a}\right), \\
C_{3}=\left(\cos \left(\frac{\pi y_{d}}{b}\right)^{2}-1\right)\left(\cos \left(\frac{\pi x_{d}}{a}\right)^{2}-1\right), \\
C_{4}=64\left(\left(\frac{L_{c_{2}} \pi^{8}\left(a^{2}+b^{2}\right)^{2} D_{2}}{64}+a^{4} b^{4} c_{0}^{2} \rho_{0}\right) D_{1}+D_{2} a^{4} b^{4} c_{0}^{2} \rho_{0}\right) \\
\times \sin \left(\frac{\pi y_{f}}{b}\right) \sin \left(\frac{\pi x_{f}}{a}\right) \sin \left(\frac{\pi y_{s}}{b}\right) \sin \left(\frac{\pi x_{s}}{a}\right) .
\end{gathered}
$$

In Eq. (B.1)-(B.4), the locations of the excitation point force, the active vibration isolation unit and the sensor are defined by the points $\left(x_{f}, y_{f}\right),\left(x_{d}, y_{d}\right)$ and $\left(x_{s}, y_{s}\right)$, respectively. 


\section{Appendix C. Bordering frequencies $\boldsymbol{\Omega}_{a}, \boldsymbol{\Omega}_{b}, \boldsymbol{\Omega}_{c}$ and $\boldsymbol{\Omega}_{d}$}

The bordering frequency, introduced in subsection 3.3.1, can be calculated by differentiating the absolute value of the transfer mobility, Eq. 28) (i.e., Eq. (35) in non-dimensional form), with respect to $c_{p}(i . e ., \eta)$. The resulting derivative is then equated to zero.

By using the non-dimensional form, the operations yield,

$$
\left(2 \Omega^{4}-(4 \alpha(\mu+1)+2 \beta+2) \Omega^{2}+4 \alpha(\mu \beta+1)+2 \beta\right)\left(\Omega^{2}-1\right)\left(\Omega^{2}-\beta\right) \Omega^{3} \eta \mu^{2}=0
$$

The four positive roots of Eq. [C.1] are the following,

$$
\Omega_{a}=1,
$$

$$
\begin{gathered}
\Omega_{b}=\frac{1}{2} \sqrt{2} \sqrt{2 \alpha(\mu+1)+\beta+1-\sqrt{4 \alpha^{2} \mu^{2}-4 \alpha \mu(\beta-2 \alpha-1)+(\beta-1+2 \alpha)^{2}}}, \\
\Omega_{c}=\sqrt{\beta}, \\
\Omega_{d}=\frac{1}{2} \sqrt{2} \sqrt{2 \alpha(\mu+1)+\beta+1+\sqrt{4 \alpha^{2} \mu^{2}-4 \alpha \mu(\beta-2 \alpha-1)+(\beta-1+2 \alpha)^{2}}} .
\end{gathered}
$$

The expressions for $\Omega_{a}$, Eq. (C.2), and $\Omega_{c}$, Eq. (C.4), are valid when $\beta>1$. When $\beta<1, \Omega_{a}$ and $\Omega_{c}$ switch places.

\section{Appendix D. Mode shapes of the full order model}

In Fig. D.21 the mode shapes of the full order model, presented in Section 2.2 and analysed in Section 4 are plotted for frequency up to $655 \mathrm{~Hz}$ in the case of $\beta<1$.

In Fig. D.21 it can be seen that for the case a, $135 \mathrm{~Hz}$, to the case e, $324 \mathrm{~Hz}$, the modes of the structural-acoustic coupled model, Fig. 1. are controlled by the vibration of the two flexible panels acoustically coupled with the air in cavity $c_{1}$ between them. Therefore, the mode shapes at these frequencies are similar to the modes of an acoustically coupled double panel with simply supported edges which couples to the $(0,0,0)$ mode of the back cavity. On the other side, the mode shown in Fig. D.21.g at $606 \mathrm{~Hz}$ seems to be controlled by the acoustic mode of the cavity $c_{2}$, such that the plate modes are distorted accordingly. At higher frequency, the mode shapes start to be more complex due to the complicated coupling between the two panels and the air in the two cavities, so it can no longer be distinguished which medium predominately governs the shape of the modes.

\section{References}

[1] J. S. Mixson, Aeroacoustics of Flight Vehicles: Theory and Practice. Volume 2: Noise Control, Tech. Rep. 1 (1991).

[2] J. F. Wilby, Aircraft interior noise, Journal of Sound and Vibration 190 (3) (1996) 545-564. doi:10.1006/jsvi. 1996.0078

[3] M. Bull, Wall-Pressure Fluctuations Beneath Turbulent Boundary Layers: Some Reflections on Forty Years of Research, Journal of Sound and Vibration 190 (3) (1996) 299-315.

[4] G. M. Corcos, The Structure of the Turbulent Pressure Field in Boundary-Layer Flows, Journal of Fluid Mechanics 18 (03) (1964) 353-378. doi:doi:10.1017/S002211206400026X 


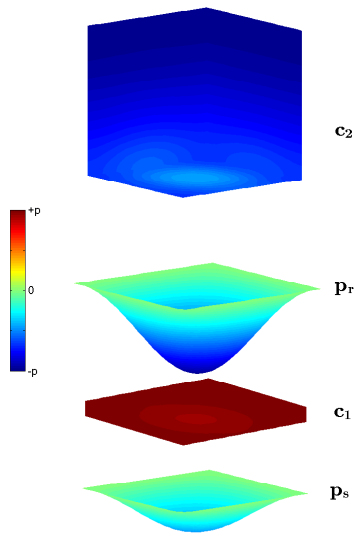

(a)

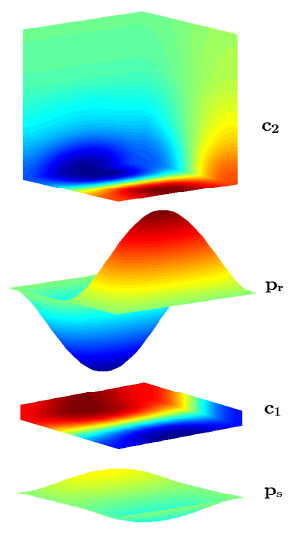

(d)

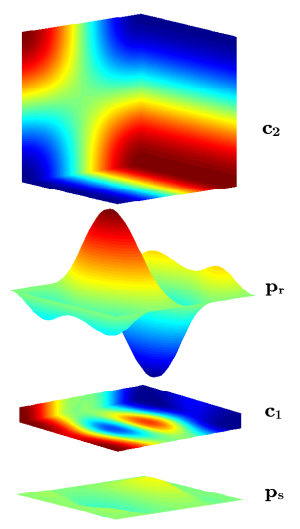

(g)

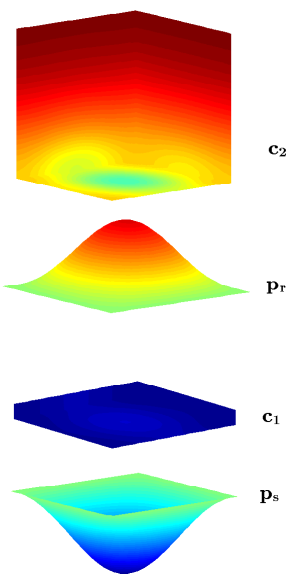

(b)

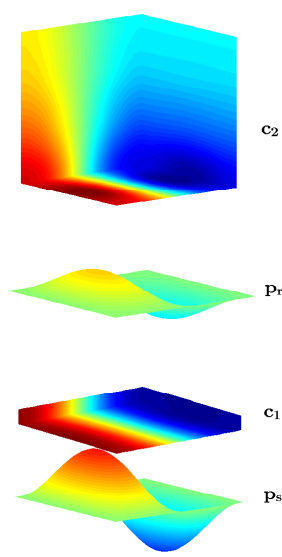

(e)

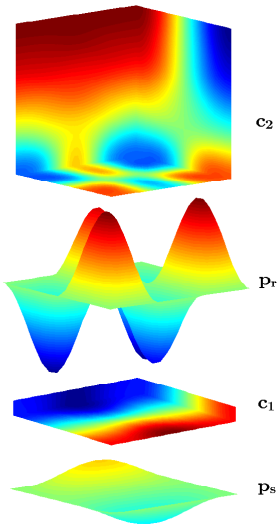

(3)

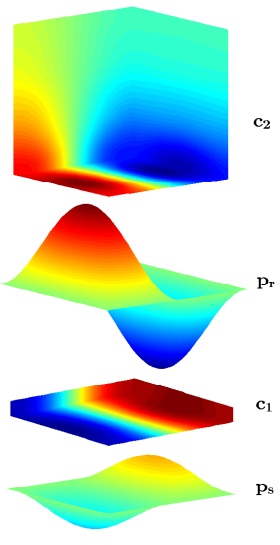

(c)

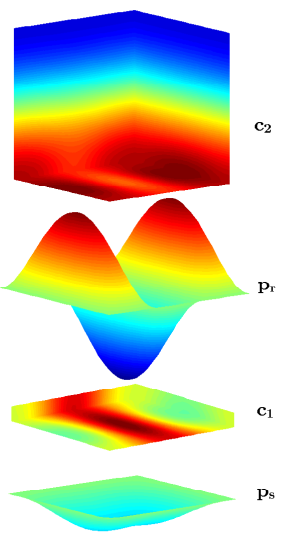

(f)
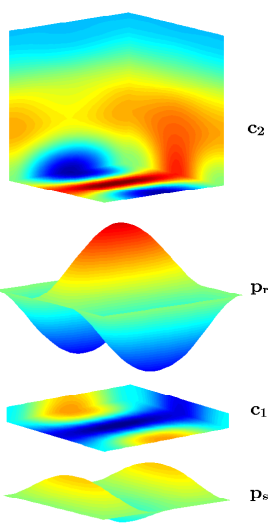

(i)

Figure D.21: Mode shapes of the full order model: $135 \mathrm{~Hz}$ (a); $201 \mathrm{~Hz}$ (b); $213 \mathrm{~Hz}$ (c); $304 \mathrm{~Hz}$ (d); $324 \mathrm{~Hz}$ (e); $411 \mathrm{~Hz}$ (f); $606 \mathrm{~Hz}(\mathrm{~g}) ; 630 \mathrm{~Hz}(\mathrm{~h}) ; 655 \mathrm{~Hz}$ (i). 
[5] William K. Blake, Mechanics of flow-induced sound and vibration. Volume II: Complex flow-structure interactions, 1986.

[6] W. K. Bonness, J. B. Fahnline, P. D. Lysak, M. R. Shepherd, Modal forcing functions for structural vibration from turbulent boundary layer flow Journal of Sound and Vibration 395 (2017) 224-239. doi : 10.1016/j • jsv. 2017. 02.023

URL http://dx.doi.org/10.1016/j.jsv.2017.02.023

[7] A. Caiazzo, R. D’Amico, W. Desmet, A Generalized Corcos model for modelling turbulent boundary layer wall pressure fluctuations, Journal of Sound and Vibration 372 (2016) 192-210. doi:10.1016/j • jsv .2016.02.036

[8] Y. Hwang, W. Bonness, S. Hambric, On Modeling Structural Excitations by Low Speed Turbulent Boundary Layer Flows, Tech. rep., The Pennsylvania State University (2003).

[9] S. H. Ko, A. H. Nuttall, Analytical evaluation of flush-mounted hydrophone array response to the Corcos turbulent wall pressure spectrum, J. Acoust. Soc. Am. 90 (1991) 579.

[10] W. W. Willmarth, F. W. Roos, Resolution and structure of the wall pressure field beneath a turbulent boundary layer, Journal of Fluid Mechanics 22 (01) (1965) 81-94

[11] F. Fahy, P. Gardonio, Sound and Structural Vibration, 2nd Edition, Elsevier, 2007.

[12] J.-H. Ho, A. P. Berkhoff, Flat acoustic sources with frequency response correction based on feedback and feedforward distributed control, The Journal of the Acoustical Society of America 137 (4) (2015) 2080-2088. doi: 10.1121/1.4914997

[13] P. Sas, C. Bao, F. Augusztinovicz, W. Desmet, Active control of sound transmission through a double panel partition, Journal Of Sound And Vibration 180 (4) (1995) 609-625. doi:10.1006/jsvi.1995.0102

[14] P. Gardonio, N. Alujević, Double panel with skyhook active damping control units for control of sound radiation, Journal of the Acoustical Society of America 128 (3) (2010) 1108-1117. doi:10.1121/1.3467772

[15] S. Elliott, P. Nelson, I. Stothers, C. Boucher, In-flight experiments on the active control of propeller-induced cabin noise, Journal of Sound and Vibration 140 (2) (1990) 219-238.

[16] S. J. Elliott, I. M. Stothers, P. A. Nelson, A Multiple Error LMS Algorithm and Its Application to the Active Control of Sound and Vibration, IEEE Transactions on Acoustics, Speech, and Signal Processing ASSP-35 (1987) 1423-1434.

[17] J. M. Wesselink, A. P. Berkhoff, Fast affine projections and the regularized modified filtered-error algorithm in multichannel active noise control., The Journal of the Acoustical Society of America 124 (2) (2008) 949-60. doi:10.1121/1.2945169

[18] J. Rohlfing, P. Gardonio, Ventilation duct with concurrent acoustic feed-forward and decentralised structural feedback active control, Journal of Sound and Vibration 333 (3) (2014) 630-645. doi:10.1016/j.jsv.2013.09. 022

[19] M. Misol, T. Haase, H. Monner, M. b. Sinapius, Causal feedforward control of a stochastically excited fuselage structure with active sidewall panel, Journal of the Acoustical Society of America 136 (4) (2014) 1610-1618. doi:10.1121/1.4895710

[20] N. Alujević, P. Gardonio, K. D. Frampton, Smart Double Panel for the Sound Radiation Control : blended velocity feedback, AIAA JOURNAL 49 (6) (2011) 1123-1134. doi:10.2514/1.44284

[21] P. Gardonio, M. Zilletti, Sweeping tuneable vibration absorbers for low-mid frequencies vibration control, Journal of Sound and Vibration 354 (2015) 1-12. doi:10.1016/j.jsv.2015.05.024

[22] N. Alujević, G. Zhao, B. Depraetere, P. Sas, B. Pluymers, W. Desmet, H2 optimal vibration control using inertial actuators and a comparison with tuned mass dampers, Journal of Sound and Vibration 333 (2014) 4073-4083. doi:10.1016/j.jsv.2014.04.038

[23] A. Preumont, A. François, F. Bossens, A. Abu-Hanieh, Force feedback versus acceleration feedback in active vibration isolation, Journal of Sound and Vibration 257 (4) (2002) 605-613. doi:10.1006/jsvi.2002.5047

[24] N. Alujević, H. Wolf, P. Gardonio, I. Tomac, Stability and performance limits for active vibration isolation using blended velocity feedback, Journal of Sound and Vibration 330 (21) (2011) 4981-4997. doi:10.1016/j.jsv. 2011.05 .020

[25] N. Alujević, H. Wolf, B. Depraetere, G. Zhao, Z. Domazet, B. Pluymers, W. Desmet, Self-tuneable velocity feedback for active isolation of random vibrations in subcritical two degree of freedom systems, Acta Acustica united with Acustica 101 (5) (2015) 950-963. doi:10.3813/AAA.918890

[26] A. Preumon, Vibration Control of Active Structures, Vol. 179, Springer Netherlands, 2011. doi:10.1007/ 978-94-007-2033-6

[27] K. DD, C. MJ, H. RA, Vibration Control Using Semi-Active Force Generators. ASME., Journal of Engineering for Industry 96 (1974) 619-626. doi:10.1115/1.3438373

[28] W. K. Bonness, Low wavenumber turbulent boundary layer wall pressure and shear stress measurements from vibration data on a cylinder in pipe flow., Ph.D. thesis, Pennsylvania State University (2009).

[29] W. R. Graham, A Comparison of Models for the Wavenumber - Frequency Spectrum of Turbulent Boundary Layer Pressures, Journal of Sound and Vibration 206 (4) (June 1995) (1997) 541-565. 
[30] F. P. Munich, F. G. R. Wien, J. S. Palaiseau, Noise Sources in Turbulent Shear Flows, Springer, 2013.

[31] G. M., Empirical spectral model of surface pressure fluctuations, Journal AIAA 42 (2004) 1788-1794.

[32] Y. F. Hwang, W. K. Bonness, S. a. Hambric, Comparison of semi-empirical models for turbulent boundary layer wall pressure spectra, Journal of Sound and Vibration 319 (1-2) (2009) 199-217. doi:10.1016/j.jsv.2008. 06.002

[33] W. R. Graham, Boundary layer induced noise in aircraft, Tech. rep., Cambridge University Engineering Department, CUED/A-AERO/TR18. (1992)

[34] C. Maury, P. Gardonio, S. J. Elliott, Model for Active Control of Flow-Induced Noise Transmitted Through Double Partitions, AIAA JOURNAL 40 (6) (2002) 1113-1121.

[35] J. Rocha, A. Suleman, F. Lau, Prediction of turbulent boundary layer induced noise in the cabin of a BWB aircraft, Shock and Vibration 19 (2012) 693-705. doi:10.3233/SAV-2011-0660

[36] J. Rocha, A. Suleman, F. Lau, Prediction of Flow-Induced Noise in Transport Vehicles: Development and Validation of a Coupled Structural-Acoustic Analytical Framework, Journal of Canadian Acoustical Association 37 (2009) 13-29.

[37] E. H. Dowell, G. F. Gorman, D. A. Smith, Acoustoelasticity: general theory, acoustic natural modes and forced response to sinusoidal excitation, including comparisons with experiment, Journal of Sound and Vibration 52 (4) (1977) 519-542.

[38] Mark J. Balas, Direct Velocity Feedback Control of Large Space Structures, Journal of Guidance, Control, and Dynamics 2 (3) (1979) 252-253.

[39] D. Newland, An Introduction to Random Vibrations, Spectral \& Wavelet Analysis, 3rd Edition, Mineola, NewYork, 1975. 